\title{
ELETROFIAÇÃO APLICADA AO DESENVOLVIMENTO DE NANOFIBRAS ADSORVENTES VISANDO O APRIMORAMENTO DE TÉCNICAS DE (MICRO)EXTRAÇÃO EM FASE SÓLIDA
}

Camilla Fonseca Silva ${ }^{a}$, Daiane D. Moraes de Paula ${ }^{a}$ e Keyller Bastos Borges ${ }^{\text {a*, (D) }}$

aDepartamento de Ciências Naturais, Universidade Federal de São João del-Rei, Campus Dom Bosco, 36301-160 São João del Rei - MG, Brasil

Recebido em 18/11/2020; aceito em 26/03/2021; publicado na web em 26/04/2021

\begin{abstract}
ELECTROSPINNING APPLIED TO THE DEVELOPMENT OF ADSORBENT NANOFIBERS AIMING AT IMPROVING SOLID PHASE (MICRO)EXTRACTION TECHNIQUES. The limitations and challenges associated with the complex analyte-matrixinterfering interactions make the development of new sorbent materials an attractive line in several research areas, ranging from the synthesis and characterization to the application of these materials. Among the different strategies for manufacturing sorbent nanomaterials, the electrospinning of nanofibers based on polymeric solutions incorporated into Metal-Organic Frameworks (MOFs), Molecularly Imprinted Polymers (MIPs) and Nanoparticles (NPs) have represented in recent years, one of the methods more versatile and promising with regard to obtaining materials with large surface area and potential for functionalization and chemical modification. In this sense, the objective of this contribution is to highlight recent work aimed at the synthesis of electrospun nanofibers applied in adsorption and sample preparation processes, highlighting the synthetic nanofiber strategies based on MOFs, MIPs and NPs and the main challenges and perspectives of the applications, although little explored, of these materials in solid phase extraction and microextraction techniques.
\end{abstract}

Keywords: electrospinning; nanofibers; sample preparation; adsorption.

\section{INTRODUÇÃo}

Nos últimos anos, a Ciência dos Materiais se tornou um dos campos científicos mais atraentes e inovadores, principalmente no que diz respeito ao desenvolvimento de novos materiais de dimensões nanométricas, conhecidos como nanomateriais. Contudo, percebese que consideráveis esforços da comunidade científica atual têm sido empreendidos no sentido de modificar a estrutura química de materiais já conhecidos e/ou de novos materiais por meio do desenvolvimento de novas estratégias sintéticas, a fim de adequálos, ou ainda, potencializá-los para diversas aplicações, incluindo separações analíticas e bioanalíticas. ${ }^{1-3}$

A Eletrofiação (ou mais comumente conhecida, do inglês, como electrospinning) tem sido reportada na literatura como uma das tecnologias mais utilizadas para a fabricação de nanomateriais, devido às suas potencialidades como simplicidade, baixo custo e versatilidade, aliadas à capacidade de produção de nanofibras altamente porosas e de grande área superficial específica, fatores esses que tornam os materiais eletrofiados, potencialmente úteis para sorção de diversos analitos em diversos tipos de amostras. ${ }^{3}$ Em particular, tal tecnologia tem se mostrado extremamente atraente e aplicável nos últimos anos na Ciência das Separações, dado o número crescente de artigos nos últimos anos, dedicados à utilização de nanofibras eletrofiadas para aplicações em preparo de amostras, de acordo com dados da Web of Science (Figura 1).

Frente ao interesse da comunidade científica no desenvolvimento, aprimoramento e utilização da técnica de eletrofiação para produção de nanomateriais, essa revisão fornece em sua parte inicial uma visão geral da técnica, incluindo seu histórico, princípios e métodos, seguida de amplas discussões relacionadas as aplicações de nanofibras eletrofiadas em técnicas de preparo de amostras e processos de adsorção, utilizando novos materiais a base de soluções poliméricas incorporadas a outros materiais adsorventes e seletivos

*e-mail: keyller@ufsj.edu.br

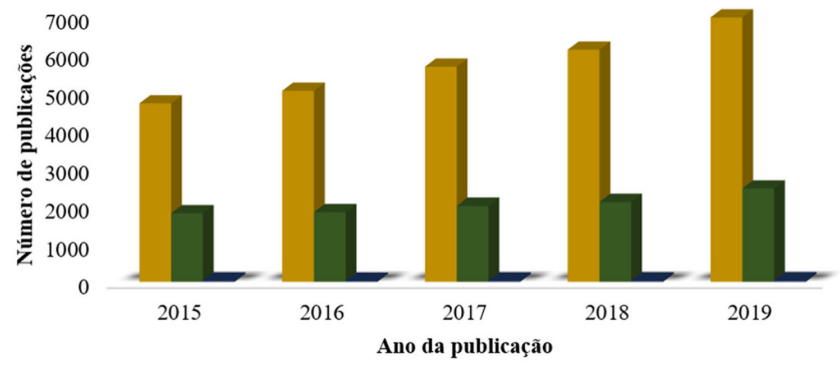

"Nanofibers $\backsim$ Nanofibers/electrospinning $₫$ Nanofibers/electrospinning/sample preparation

Figura 1. Representação esquemática do número de publicações por ano sobre nanofibras, nanofibras eletrofiadas e nanofibras eletrofiadas para preparo de amostras, nos anos de 2015 a 2019. Dados da Web of Science

convencionalmente já utilizados, como as estruturas metal-orgânicas (MOF, do inglês Metal Organic Framework), polímeros de impressão molecular (MIP, do inglês Molecularly Imprinted Polymer) e nanopartículas (NP, do inglês Nanoparticles).

\section{TÉCNICA DE ELETROFIAÇÃO}

\section{Surgimento e histórico da técnica}

Embora o termo eletrofiação tenha sido utilizado a partir dos anos 90, a origem da técnica de produção de nano ou microfibras por meio da aplicação de força eletrostática, remonta ao século XIX. ${ }^{4-13}$ $\mathrm{O}$ fenômeno observado pela primeira vez por Rayleigh, foi de fato consolidado experimentalmente por Cooley e Morton, ao descrever o método de aplicação de altas voltagens para produção de fios finos e relatar a influência da viscosidade e volatilidade da solução polimérica precursora, bem como da intensidade do campo elétrico aplicado na qualidade dos fios produzidos. ${ }^{14}$ Anos mais tarde, Zeleny evidenciou em seu trabalho um modelo matemático para tratar o comportamento das soluções quando expostas à ação de um campo elétrico externo. ${ }^{15}$ 
Contudo, somente em meados do século XX, a eletrofiação teve de fato suas bases experimentais lançadas a partir da primeira patente depositada Formhals, que relatava a produção de fios têxteis de acetato de celulose dispersos em solução de acetona e éter monometílico de etilenoglicol, ${ }^{4}$ seguido das contribuições de modelagem matemática propostas por Taylor acerca do processo de distorção e alongamento da gota de solução precursora formada na extremidade da agulha ou capilar, no formato característico de um cone (conhecido como Cone de Taylor) devido a aplicação de forças eletrostáticas. ${ }^{16}$

Desde então, diversas patentes incluindo diferentes soluções poliméricas foram arquivadas, fazendo com que a popularidade da técnica fosse alcançada no meio científico, tanto pelo interesse em estudar o processo de eletrofiação, quanto a natureza e utilidade das nanofibras produzidas. No campo específico da Ciência das Separações, a eletrofiação ganhou destaque com o interesse em produzir estruturas fibrosas de alto desempenho analítico como metais, semicondutores, polímeros, cerâmica e compósitos (modificados pré ou pós-eletrofiação), com diâmetros micrométricos ou nanométricos..$^{1-3}$

\section{O processo de eletrofiação}

O conceito de eletrofiação se refere à produção de fibras (em geral, nanométricas) formadas a partir de soluções precursoras expostas a um campo elétrico em condições de pressão e temperatura ambiente, sendo em geral, o sistema composto basicamente de quatro componentes: (i) Bomba de seringa para transporte da solução precursora; (ii) Agulha ou fieira; (iii) Fonte de energia de alta tensão (da ordem de KV) e (iv) Coletor aterrado. O sistema tipicamente é montado adotando a configuração vertical (Figura 2a) ou ainda, pode assumir a configuração horizontal, sendo esta menos comum (Figura 2b). ${ }^{17-19}$

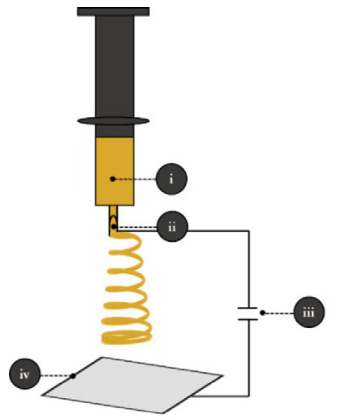

(a)

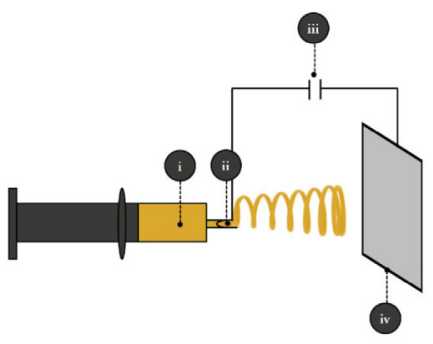

(b)
Figura 2. Diagrama esquemático de um sistema de eletrofiação com: (i) bomba de seringa; (ii) fieiralagulha; (iii) fonte de alta tensão e (iv) coletor aterrado. A alta tensão é aplicada entre a fieira e o coletor aterrado. Em (a) configuração vertical e (b) configuração horizontal

O processo consiste basicamente de três etapas sequenciais, a primeira de formação do fluxo da solução precursora, a segunda do alongamento dos fios e a terceira da solidificação da fibra. Desta forma, inicialmente ocorre a formação da gota de solução precursora na ponta da fieira (ou da agulha) seguida do seu carregamento eletrostático, sendo a etapa inicial deste carregamento marcada pelo balanço entre as forças de natureza eletrostática (atrativa em relação ao coletor) e a tensão superficial da solução (repulsiva em relação ao coletor). ${ }^{4}$ Subsequentemente, devido ao aumento da intensidade crítica do campo elétrico, até o seu valor crítico, as forças eletrostáticas superficiais da gota superam a tensão superficial que a sustenta, resultando na ejeção forçada do jato de solução do polímero precursor, no formato alongado e distorcido, denominado
Cone de Taylor, conforme apresentado na Figura 3. Por fim, ocorre a volatilização do solvente em contato com a atmosfera ambiente, de forma que o material sólido é depositado sob o coletor. ${ }^{17-19}$

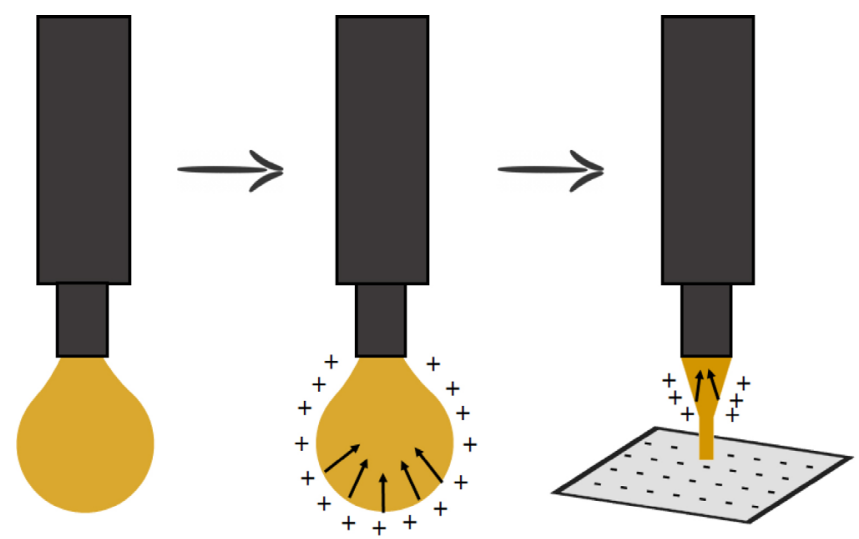

Figura 3. Esquema representativo do alongamento e distorção das gotas no formato de Cone de Taylor. As setas na gota representam a tensão superficial, os sinais positivos representam as cargas elétricas acumuladas na superfície da gota e os sinais negativos representa, as cargas elétricas acumuladas no coletor

\section{Modificações na configuração do sistema de injeção e de coleta}

De acordo com a seção anterior, as nanofibras comumente são coletadas aleatoriamente em uma placa coletora metálica ou coberta por folha de papel alumínio. Porém, para serem usadas em determinadas aplicações, o alinhamento unilateral destas pode ser crucial. A Figura 4a representa um aparato experimental com dois coletores que visam melhorar a orientação e posicionamento das fibras. ${ }^{20}$ Análogo ao sistema anterior, o primeiro coletor é aterrado, enquanto o segundo é mantido a $6 \mathrm{kV}$, o que força o polímero eletrostaticamente carregado (ver: Cones de Taylor) a saltar para o segundo coletor, produzindo fibras com alto nível de alinhamento. ${ }^{20,21}$ Além dessa, outras estratégias podem ser utilizadas para produção de nanofibras alinhadas, a exemplo do uso de um coletor aterrado em uma placa de Petri envolta por um fio fino de cobre metálico conectado a um tambor rotativo (motorizado) com velocidade de rotação controlada (Figura 4b). Neste sistema, as fibras são coletadas de forma desordenada na placa de Petri, porém adquirem ordenamento no tambor rotativo..$^{22,23}$

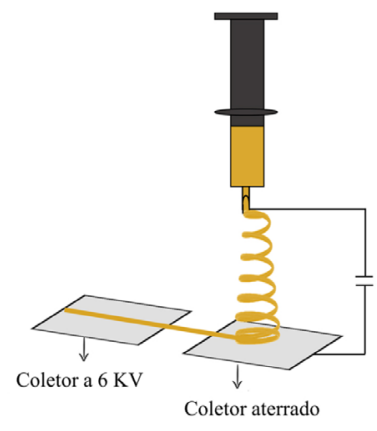

(a)

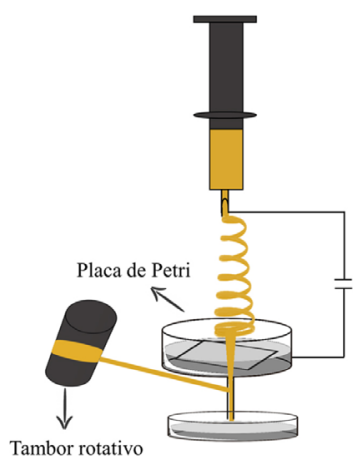

(b)
Figura 4. Esquema representativo de configurações de coleta de nanofibras. Em: (a) sistema de coletores paralelos e (b) sistema de coleta com tambor rotativo

Outras diferentes propriedades, além do alinhamento e ordenamento, podem ser obtidas por meio de simples ajustes do 
sistema operacional. No caso específico da eletrofiação coaxial, emprega-se um sistema semelhante ao da eletrofiação comum, porém, com modificações no sistema de injeção da solução precursora. Basicamente, insere-se de forma concêntrica, uma agulha de menor diâmetro interno no interior de uma agulha de diâmetro interno maior, sendo que as duas recebam de forma independente, mas simultânea, duas soluções precursoras distintas, conforme mostrado na Figura 5.24,25

A produção da nanofibra coaxial ocorre pelo mesmo mecanismo da eletrofiação comum, em que a solução precursora $\mathrm{P} 1$ passada pela agulha de menor diâmetro se deforma (formando o cone de Taylor) e a solução precursora $\mathrm{P} 2$ passada pela agulha de maior diâmetro, desenvolve-se na ponta dos cones. ${ }^{24} \mathrm{O}$ uso deste sistema permite a produção de materiais com estrutura casca-núcleo (core-shell), que apresentam propriedades diferenciadas pela combinação das propriedades de duas soluções precursoras distintas. Em relação a viscosidade, é necessário que ambas as soluções poliméricas apresentem viscosidade suficiente para ultrapassar a tensão interfacial entre as duas soluções e formar a estrutura desejada. ${ }^{24-26}$

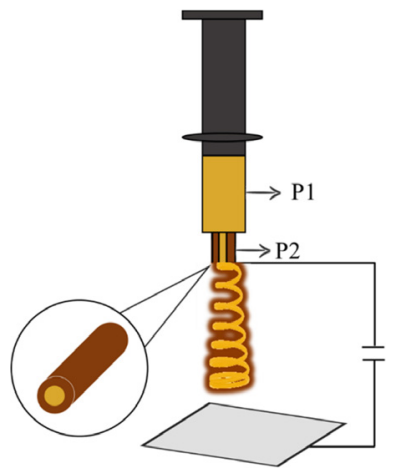

Figura 5. Esquema representativo do sistema de eletrofiação coaxial para produção de nanofibras core-shell

\section{Parâmetros de influência na eletrofiação}

Embora a técnica seja considerada versátil e eficiente para produção de diversas nanofibras, consensualmente, diversos autores relatam a necessidade de otimizar os parâmetros relacionados: (i) a natureza da solução precursora (como concentração, viscosidade, tensão superficial e condutividade); (ii) ao processamento das fibras (como intensidade do campo elétrico aplicado, vazão, diâmetro da agulha/fieira e distância do coletor) e (iii) as condições ambientais (como pressão, temperatura e umidade); a fim de se obter materiais com boa reprodutibilidade e funcionalidade.,

\section{Parâmetros da solução polimérica}

Em relação aos parâmetros da solução, fatores como concentração, viscosidade, tensão superficial (correlacionados entre si) e condutividade da solução precursora interferem diretamente na estrutura dos materiais obtidos. ${ }^{27-30} \mathrm{~A}$ variação na concentração da solução afeta a formação das fibras devido as influências em suas propriedades, como viscosidade e tensão superficial. Assim, em concentrações baixas, a consequente baixa viscosidade da solução precursora, pode ocasionar o processo de eletropulverização, no qual o jato da solução precursora divide-se em gotículas durante o transporte até o coletor, levando a formação de NPs em vez de nanofibras. Já em concentrações intermediárias, podem ser formadas misturas de NPs e nanofibras, porém com nanofibras pouco estáveis. Finalmente, em altas concentrações, em geral conduz-se a obtenção de nanofibras lisas, uniformes e sem grânulos, demonstrando a importância de se trabalhar em faixas de concentração adequadas..$^{27,28}$
Além da concentração, a influência do peso molecular da solução precursora também tem um efeito direto na viscosidade da solução. Dessa forma, ao diminuir o peso molecular da solução pode haver a formação de grânulos na fibra, ao passo que, ao aumentar o peso molecular, há uma maior tendência de obtenção de fibras lisas e uniformes. ${ }^{29}$ Já em relação a condutividade, em geral o seu aumento favorece a formação de fibras mais finas e uniformes, sendo que esse fator é determinado principalmente pelo tipo de precursor, tipo de solvente e adição de sal. ${ }^{30}$

\section{Parâmetros do processo}

Em relação aos parâmetros do processo, fatores como tensão aplicada, vazão da solução precursora, distância do alvo coletor e diâmetro da agulha/fieira são cruciais na produção das nanofibras. ${ }^{31-35}$ A variação da tensão aplicada é o fator crucial, pois o material só será ejetado no sentido do coletor se a tensão aplicada for maior do que a tensão limite. Ao se aplicar valores mais altos de tensão, verifica-se um aumento das forças repulsivas eletrostáticas, levando a consequente diminuição do diâmetro da nanofibra ou em alguns casos, a formação de NPs. ${ }^{31,32}$

Além do controle da tensão aplicada, recomenda-se que vazão da solução precursora dentro da seringa, tenha taxa de fluxo mais baixa, a fim de se oferecer tempo suficiente para a polarização da gota e para seu consequente alongamento, não observado em casos de operações com alta taxa de fluxo que acarretam a formação de fibras com diâmetro espesso, devido ao curto tempo de secagem (que ocorre à medida que o jato se afina) antes de atingir o coletor. ${ }^{33} \mathrm{Já}$ em relação à distância entre o coletor e a ponta da seringa, verifica-se que em casos de curtas distâncias, o tempo oferecido para a solidificação da fibra também é curto e a volatilização do solvente é apenas parcial, podendo levar a formação de NPs, enquanto no caso de longas distâncias, fibras com diâmetros muito espessos podem ser obtidas. ${ }^{34,35}$

\section{Parâmetros ambientais}

Em relação aos parâmetros ambientais, fatores como umidade e temperatura podem afetar os diâmetros e morfologias das fibras obtidas. ${ }^{30-33} \mathrm{O}$ aumento da temperatura, acarreta no aumento da taxa de evaporação do solvente, enquanto reduz a viscosidade da solução precursora e, portanto, acarreta a produção de nanofibras com diâmetros menores. Já a umidade, ao ser diminuída, acarreta o aumento da velocidade da evaporação do solvente, enquanto a alta umidade acarreta o aumento do diâmetro, fazendo com que sejam obtidas fibras mais grossas. ${ }^{36}$

\section{APLICAÇÕES DE NANOFIBRAS ELETROFIADAS EM PROCESSOS ANALÍTICOS}

Devido a versatilidade da técnica de eletrofiação, diferentes materiais, como partículas esféricas, fibras de mono ou multicamadas, podem ser obtidos e posteriormente aplicados em processos analíticos como preparo de amostras e adsorção. A redução das dimensões dos materiais eletrofiados às escalas micro ou nanométrica (com diâmetros que variam $10 \mathrm{~nm}$ a $10 \mu \mathrm{m}$ ), permitem aprimorar significativamente as propriedades químicas e mecânicas destes, além de acarretar o aumento da área superficial, e por consequência, da capacidade extratora e da cinética de interação com moléculas alvo. Aliada ao poder de sorção, a possibilidade de funcionalidade, permite a obtenção de sorventes altamente seletivos para diferentes analitos. ${ }^{37}$

Frente ao grande número de artigos e revisões que descrevem a aplicabilidade de nanofibras eletrofiadas em preparo de amostras e processos de adsorção, ${ }^{37-45}$ concentraremos nossa atenção às principais contribuições dos últimos anos relacionadas aos materiais de tendência de uso em preparo de amostras/adsorção e que têm sido 
atualmente utilizados combinados com as nanofibras poliméricas, como MOF, MIP e NP. A combinação de MOFs, MIPs e NPs com nanofibras poliméricas atribuem diferentes propriedades físicoquímicas aos materiais, gerando uma extensa classe de materiais convencionalmente chamados de nanofibras enriquecidas, aplicados extensivamente nas técnicas de extração em fase sólida (SPE, do inglês solid phase extraction) e microextração em fase sólida (SPME, do inglês solid phase microextraction).

\section{Nanofibras enriquecidas com MOFs}

As MOFs são uma classe de materiais híbridos, de alta porosidade, alta área superficial, boa estabilidade térmica e química e que têm sido frequentemente aplicados, nas últimas décadas, em técnicas de preparo de amostras. Formados por meio da coordenação covalente de íons ou clusters metálicos à ligantes orgânicos, em um tipo de interação ácido-base de Lewis, as MOFs encontram-se na classe de compostos de coordenação, cuja estrutura tridimensional apresenta ambientes favoráveis à adsorção de analitos, aliados à alta transferência de massa para técnicas de SPE (Figura 6). ${ }^{46,47}$

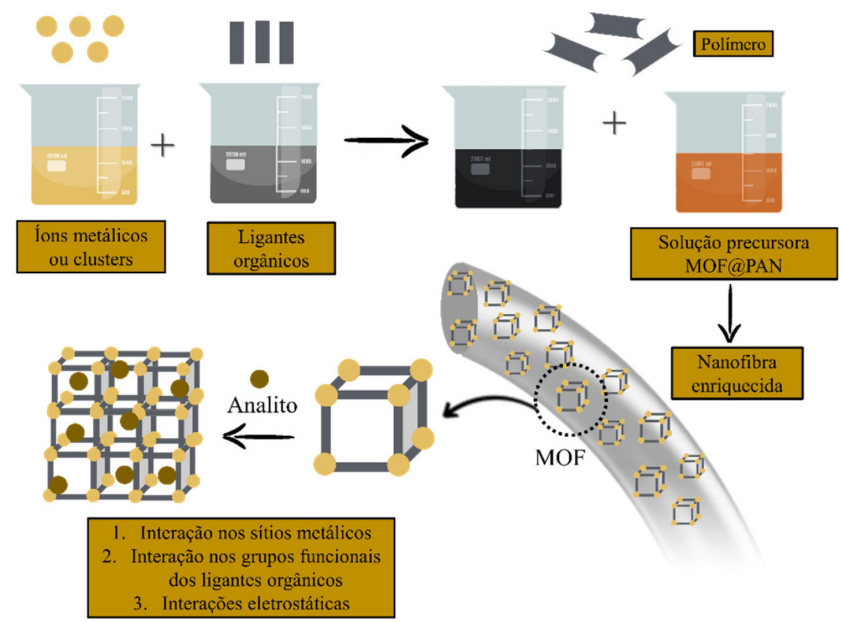

Figura 6. Ilustração esquemática da obtenção de nanofibras enriquecidas com MOF via Eletrofiação e das interações envolvidas na sorção de analitos

Consensualmente, a presença dos ligantes orgânicos, em geral ácidos aromáticos, atribuem a existência de uma cavidade hidrofóbica, de alta afinidade para compostos orgânicos apolares ou de baixa polaridade, além da possibilidade do estabelecimento de interações intermoleculares do tipo ligação de hidrogênio, empilhamento $\pi-\pi$, dipolo-dipolo e interação eletrostática, permitindo maior eficiência como sorvente em comparação com outros materiais já conhecidos. Contudo, as maiores limitações desses materiais no que tange ao uso como extrator, se referem à baixa estabilidade química em meio aquoso e básico, além da dessorção incompleta ou irreversível dos analitos, devido à forte interação MOF-analito. ${ }^{46,47} \mathrm{~A}$ Tabela 1 apresenta os principais trabalhos relatados nos últimos anos visando o desenvolvimento e utilização de nanofibras poliméricas incorporadas com MOFs para aplicações em preparo de amostras/adsorção.

Efome e colaboradores, em uma linha de três trabalhos sequenciais, produziram MOFs baseados em Fe(III) e $\mathrm{Zr}$ (IV) juntamente com os polímeros poliacrilonitrila e fluoreto de polivinilideno para adsorção de íons $\mathrm{Pb}$ (II), $\mathrm{Hg}$ (II), Cd(II) e Zn(II) em solução aquosa. ${ }^{48,49} \mathrm{Em}$ todos os trabalhos, os autores relataram a alta capacidade de adsorção, o excelente desempenho de filtração e regeneração das membranas, além da alta estabilidade em meio aquoso com potencial uso para tratamento de água, sendo possível uma produção superior a $500 \mathrm{~L}$ de água pura por $\mathrm{m}^{2}$ de membrana. Nesse mesmo sentido, Asiabi e col. relataram a utilização de nanofibras a base de $\mathrm{Al}$ (III) e quitosana, utilizadas como material adsorvente na técnica de SPE visando a extração de íons $\mathrm{Pb}$ (II) e $\mathrm{Cd}(\mathrm{II})$ em amostras de moluscos, tendo sido otimizados parâmetros como $\mathrm{pH}$ das amostras, quantidade de sorvente e concentração, tipo e volume de eluente. $\mathrm{O}$ material sintetizado apresentou excelentes propriedades, como grande área superficial e capacidade de adsorção superior às MOFs e quitosana isoladas, dada a combinação das vantagens do material não poroso inorgânico e do polímero orgânico. ${ }^{51}$

Visando a extração de aldeídos e estrogênios em urina, Liu e col. e Asiabi e col., respectivamente, relataram a utilização de nanofibras enriquecidas com MOF a base de $\mathrm{Cu}$ (II) e $\mathrm{Zn}$ (II). Liu e col. aplicaram o material sintetizado na técnica de microextração acoplada a cromatografia líquida de alta eficiência e otimizou parâmetros como a taxa de amostragem, taxa de eluição e natureza e volume do solvente de dessorção para seis diferentes aldeídos. A nanofibra eletrofiada desenvolvida por Liu e col. apresentou alta porosidade, grande área superficial, alta estabilidade e hidrofobicidade, além de excelente eficiência de extração. ${ }^{52}$ Já as nanofibras sintetizadas por Asiabi e col. foram empacotadas em cartuchos de SPE, tendo sido também otimizados os parâmetros de eficiência na extração, como $\mathrm{pH}$, força iônica, volume de amostra e reutilização do adsorvente, tendo sido obtidas recuperações de diferentes fármacos em amostras de urina na faixe de 82,8 a $94,8 \% .{ }^{53}$

Nanofibras à base de $\mathrm{Fe}(\mathrm{II})$ e $\mathrm{Cu}$ (II) foram aplicadas com sucesso em SPE por Amini e col., Asiabi e col., e Arabsorkhi e col. visando a determinação de fármacos benzodiazepínicos como o nitrazepam e oxazepam, do canabinóide THC e do antibiótico tetraciclina em amostras de plasma, sendo que Amini e colaboradores utilizaram a técnica de SPE adaptada em ponteira (do inglês, Pipette Tip Solid Phase Extraction, PT-SPE) e os demais autores usaram a SPE tradicional. ${ }^{59,61,62}$ Os autores reportaram recuperações de benzodiazepínicos em cerca de 92 a $100 \%$, de THC de $88,2 \%$ a $92,4 \%$ e de tetraciclina de 97,3 a $104,5 \%$. De forma análoga, a produção das nanofibras enriquecidas com MOFs se mostrou adequada para extração de sulfonamidas em amostras de carne animal, conforme relatado por Yan e col. A nanofibra eletrofiada foi aplicada como um adsorvente promissor na técnica de PT-SPE, com recuperações variando de 86 a $111 \%$. $^{64}$

\section{Nanofibras enriquecidas com MIPs}

Os MIPs são uma classe de materiais poliméricos, de alta afinidade e seletividade para moléculas alvo, devido à presença de cavidades seletivas em sua estrutura, cujo tamanho, forma e funcionalidade química apresentam-se complementares à molécula molde (MM) utilizada no processo de polimerização, junto aos monômeros funcionais (MFs), espacialmente organizados ao redor da MM, por meio do estabelecimento de ligações covalentes ou mais comumente, interações intermoleculares. A cavidade gerada após a eluição da MM por extração com solvente orgânico apropriado ou clivagem química, atribui ao material os sítios específicos de reconhecimento, favoráveis à retenção do analito em posteriores identificações e quantificações (Figura 7). ${ }^{67-69}$

Dessa forma, a abordagem de impressão molecular durante a eletrofiação, embora complexa devido às discrepâncias entre as técnicas, é alcançada por meio da introdução do polímero impresso pré-formado nas matrizes poliméricas a serem eletrofiadas. Contudo, a baixa reticulação tem sido relatada como a maior limitação desses materiais no que tange ao uso como extrator, devido às mudanças estruturais das cavidades seletivas. Alternativamente à reticulação clássica promovida na síntese MIPs, exploram-se as interações entre as principais cadeias poliméricas não reticuladas, além das 
Tabela 1. Nanofibras de MOFs reportadas na literatura para aplicações em adsorção e/ou preparo de amostras em diferentes matrizes

\begin{tabular}{|c|c|c|c|c|}
\hline Analito & Material & Amostra & Condições da eletrofiação & Ref. \\
\hline Íons $\mathrm{Pb}(\mathrm{II})$ e $\mathrm{Hg}(\mathrm{II})$ & $\begin{array}{c}\text { MOF-808/ } \\
\text { MOF-F300@PAN/ } \\
\text { PVDF }\end{array}$ & Solução aquosa & $\begin{array}{c}\text { Vazão: } 0,10-0,15 \mathrm{~mm} \mathrm{~min}^{-1} \\
\text { Tensão: } 15-20 \mathrm{KV} \\
\text { Distância do coletor: } 15 \mathrm{~cm} \\
\text { Tipo de coletor: folha de papel alumínio } \\
\mathrm{T}=25^{\circ} \mathrm{C} \text { umidade }=40 \%\end{array}$ & 48 \\
\hline Íons Cd(II) e Zn (II) & MOF-808@PAN & Solução aquosa & $\begin{array}{c}\text { Vazão: } 0,15 \mathrm{~mm} \mathrm{m^{-1 }} \\
\text { Tensão: } 15 \mathrm{KV} \\
\text { Distância do coletor: } 15 \mathrm{~cm} \\
\text { Tipo de coletor: tambor rotativo coberto por } \\
\text { papel alumínio }(140 \mathrm{rpm}) \\
\mathrm{T}=25^{\circ} \mathrm{C} \text { umidade }=40 \%\end{array}$ & 49 \\
\hline $\begin{array}{l}\text { Íons } \mathrm{Zn}(\mathrm{II}), \mathrm{Cd}(\mathrm{II}) \\
\mathrm{Pb}(\mathrm{II}) \text { e } \mathrm{Hg}(\mathrm{II})\end{array}$ & MOF-808@PAN & Solução aquosa & $\begin{array}{c}\text { Vazão: } 0,11-0,15 \mathrm{~mm} \mathrm{~min}^{-1} \\
\text { Tensão: } 15-20 \mathrm{KV} \\
\text { Distância do coletor: } 15 \mathrm{~cm} \\
\text { Tipo de coletor: folha de papel alumínio } \\
\mathrm{T}=25^{\circ} \mathrm{C} \text { umidade }=40 \% \\
\end{array}$ & 50 \\
\hline Íons $\mathrm{Pb}(\mathrm{II})$ e $\mathrm{Cd}(\mathrm{II})$ & MIL-68(Al)@quitosana & Moluscos & $\begin{array}{c}\text { Vazão: } 2,0 \mathrm{~mL} \mathrm{~h}^{-1} \\
\text { Tensão: } 17 \mathrm{KV} \\
\text { Distância do coletor: } 12 \mathrm{~cm} \\
\text { Tipo de coletor: folha de papel alumínio } \\
\text { Temperatura ambiente } \\
\end{array}$ & 51 \\
\hline Aldeídos & MOF-199@PS & Urina humana & $\begin{array}{c}\text { Vazão: } 0,8 \mathrm{~mL} \mathrm{~h}^{-1} \\
\text { Tensão: } 20 \mathrm{KV} \\
\text { Distância do coletor: } 20 \mathrm{~cm} \\
\text { Tipo de coletor: folha de papel alumínio } \\
\text { Temperatura ambiente }\end{array}$ & 52 \\
\hline Estrogênios & CH $\mathrm{H}_{3} \mathrm{MOF}-5 @ \mathrm{PAN}$ & Urina & $\begin{array}{c}\text { Vazão: } 2,0 \mathrm{~mL} \mathrm{~h}^{-1} \\
\text { Tensão: } 18 \mathrm{KV} \\
\text { Distância do coletor: } 12 \mathrm{~cm} \\
\text { Tipo de coletor: folha de papel alumínio } \\
\text { Temperatura ambiente } \\
\end{array}$ & 53 \\
\hline Pesticida organofosforado & ZIF-67@PAN/Co $\left(\mathrm{CH}_{3} \mathrm{COO}\right)_{2}$ & $\begin{array}{l}\text { Águas residuais } \\
\text { agrícolas }\end{array}$ & $\begin{array}{c}\text { Vazão: } 0,5 \mathrm{~mL} \mathrm{~h}^{-1} \\
\text { Tensão: } 11 \mathrm{KV} \\
\text { Distância do coletor: } 16 \mathrm{~cm} \\
\text { Tipo de coletor: folha de papel alumínio } \\
\text { Temperatura ambiente } \\
\end{array}$ & 54 \\
\hline Glicose & Ni-CoO@PAN/SDS & $\begin{array}{l}\text { Solução padrão } \\
\text { de glicose }\end{array}$ & $\begin{array}{c}\text { Vazão: } 0,5 \mathrm{~mL} \mathrm{~h}^{-1} \\
\text { Tensão: } 15 \mathrm{KV} \\
\text { Distância do coletor: } 15 \mathrm{~cm} \\
\text { Tipo de coletor: folha de papel alumínio } \\
\text { Temperatura ambiente }\end{array}$ & 55 \\
\hline Aldeído e aditivo alimentar & MIL-100(Fe)@PAN & Águas residuais & $\begin{array}{c}\text { Vazão: } 0,8 \mathrm{~mL} \mathrm{~h}^{-1} \\
\text { Tensão: } 16 \mathrm{KV} \\
\text { Distância do coletor: } 20 \mathrm{~cm} \\
\text { Tipo de coletor: tambor rotativo } \\
\text { Temperatura ambiente }\end{array}$ & 56 \\
\hline $\begin{array}{l}\text { Gases } \mathrm{Cl}_{2}, \mathrm{NO}_{2} \text { e agentes } \\
\text { de guerra }\end{array}$ & UiO-66-NH $\mathrm{NH}_{2} @ \mathrm{PS}$ & Mistura de gases & $\begin{array}{c}\text { Vazão: } 3,0 \mathrm{~mL} \mathrm{~h}^{-1} \\
\text { Tensão: } 12 \mathrm{KV} \\
\text { Tipo de coletor: tambor rotativo }(300 \mathrm{rpm})\end{array}$ & 57 \\
\hline Clorpirifós & Amino-Zr-MOF@PAN & $\begin{array}{l}\text { Águas de rios e } \\
\text { águas residuais }\end{array}$ & $\begin{array}{c}\text { Vazão: } 2,0 \mathrm{~mL} \mathrm{~h}^{-1} \\
\text { Tensão: } 18 \mathrm{KV} \\
\text { Distância do coletor: } 15 \mathrm{~cm} \\
\text { Tipo de coletor: folha de papel alumínio } \\
\text { Temperatura ambiente } \\
\end{array}$ & 58 \\
\hline Nitrazepam e Oxazepam & MIL-53(Fe)@PAN & $\begin{array}{l}\text { Solução aquosa, } \\
\text { urina e plasma }\end{array}$ & $\begin{array}{c}\text { Vazão: } 2,0 \mu \mathrm{L} \mathrm{min}{ }^{-1} \\
\text { Tensão: } 17 \mathrm{KV} \\
\text { Distância do coletor: } 15 \mathrm{~cm} \\
\text { Tipo de coletor: folha de papel alumínio } \\
\text { Temperatura ambiente }\end{array}$ & 59 \\
\hline $\begin{array}{l}\text { Azul de metileno e vermelho } \\
\text { do congo }\end{array}$ & $\begin{array}{l}\text { ZIF-8(Au)@PAN e } \\
\text { ZIF-8(Ni)@PAN }\end{array}$ & Solução padrão & $\begin{array}{c}\text { Vazão: } 15 \mu \mathrm{L} \mathrm{min}{ }^{-1} \\
\text { Tensão: } 12 \mathrm{KV} \\
\text { Distância do coletor: } 15 \mathrm{~cm} \\
\text { Tipo de coletor: folha de papel alumínio } \\
\text { Temperatura ambiente }\end{array}$ & 60 \\
\hline
\end{tabular}


Tabela 1. Nanofibras de MOFs reportadas na literatura para aplicações em adsorção e/ou preparo de amostras em diferentes matrizes (cont.)

\begin{tabular}{|c|c|c|c|c|}
\hline Analito & Material & Amostra & Condições da eletrofiação & Ref \\
\hline THC & MIL-101(Fe)@quitosana & Sangue total & $\begin{array}{c}\text { Vazão: } 2,0 \mathrm{~mL} \mathrm{~h}^{-1} \\
\text { Tensão: } 18 \mathrm{KV} \\
\text { Distância do coletor: } 15 \mathrm{~cm} \\
\text { Tipo de coletor: folha de papel alumínio } \\
\text { Temperatura ambiente }\end{array}$ & 61 \\
\hline Tetraciclina & Cu-BTC@PAN & Plasma & $\begin{array}{c}\text { Vazão: } 0,3 \mathrm{~mL} \mathrm{~h}^{-1} \\
\text { Tensão: } 18 \mathrm{KV} \\
\text { Distância do coletor: } 15 \mathrm{~cm} \\
\text { Tipo de coletor: folha de papel alumínio } \\
\text { Temperatura ambiente } \\
\end{array}$ & 62 \\
\hline Bisfenol-A & ZIF-8@PAN & $\begin{array}{c}\text { Águas residuais e águas } \\
\text { de rios }\end{array}$ & $\begin{array}{c}\text { Vazão: } 0,8 \mathrm{~mL} \mathrm{~h}^{-1} \\
\text { Tensão: } 19 \mathrm{KV} \\
\text { Distância do coletor: } 15 \mathrm{~cm} \\
\text { Tipo de coletor: folha de papel alumínio } \\
\text { Temperatura ambiente } \\
\end{array}$ & 63 \\
\hline Sulfonamidas & SNW-1@PAN & Carne & $\begin{array}{c}\text { Vazão: } 1,0 \mathrm{~mL} \mathrm{~h}^{-1} \\
\text { Tensão: } 19 \mathrm{KV} \\
\text { Distância do coletor: } 20 \mathrm{~cm} \\
\text { Tipo de coletor: tambor rotativo } \\
(300 \mathrm{rpm}) \\
\text { Temperatura ambiente, umidade }=30 \%\end{array}$ & 64 \\
\hline Dióxido de carbono & MOF-199@PAN & Gás & $\begin{array}{c}\text { Vazão: } 1,2-1,4 \mathrm{~mL} \mathrm{~h}^{-1} \\
\text { Tensão: } 25-26 \mathrm{KV} \\
\text { Tipo de coletor: botijão rotativo cilíndrico poroso } \\
(120-140 \mathrm{rpm}) \\
\text { Temperatura ambiente } \\
\text { Umidade }=30-40 \%\end{array}$ & 65 \\
\hline Íon $\mathrm{Cu}$ (II) & ZIF-8@PAN & Solução padrão & $\begin{array}{c}\text { Vazão: } 0,2 \mathrm{~mm} \mathrm{~min}{ }^{-1} \\
\text { Tensão: } 28 \mathrm{KV} \\
\text { Distância do coletor: } 12 \mathrm{~cm}\end{array}$ & 66 \\
\hline
\end{tabular}

MOF-808 = $\left[\mathrm{Zr}_{6} \mathrm{O}_{4}(\mathrm{OH})_{4}(\mathrm{COOH})_{6}(\mathrm{BTC})_{2}\right] ;$ MOF-F300 = $\left[\mathrm{Fe}_{3} \mathrm{OF}-\left(\mathrm{H}_{2} \mathrm{O}\right)_{2}(\mathrm{BTC})_{2} \mathrm{nH}_{2} \mathrm{O}\right] ;$ PAN = poliacrilonitrila; PVDF= fluoreto de polivinilideno; MOF-199 = $\left[\mathrm{Cu}_{3}(\mathrm{BTC})_{2}\right] ; \mathrm{PS}=$ poliestireno; UiO-66 $=\left[\mathrm{Zr}_{6} \mathrm{O}_{4}(\mathrm{OH})_{4}\right] ;$ MOF-5 = $\mathrm{Zn}_{4} \mathrm{O}(\mathrm{BDC})_{3} ; \mathrm{ZIF}-67=\left(\left[\mathrm{Co}(\mathrm{MeIm})_{2}\right]_{\mathrm{n}}\right) ; \mathrm{SDS}=$ dodecilsulfato de sódio; MIL-100(Fe) $=\left[\mathrm{Fe}_{3} \mathrm{X}\left(\mathrm{H}_{2} \mathrm{O}\right)_{2} \mathrm{O}\left[\left(\mathrm{C}_{6} \mathrm{H}_{3}\right)-\left(\mathrm{CO}_{2}\right)_{3}\right]_{2} \cdot \mathrm{nH}_{2} \mathrm{O}\right] ; \mathrm{MIL}-53(\mathrm{Fe})=\left[\mathrm{Fe}(\mathrm{OH})\left(\mathrm{O}_{2} \mathrm{C}-\mathrm{C}_{6} \mathrm{H}_{4}-\mathrm{CO}_{2}\right)\right] ; \mathrm{ZIF}-8=\left[\mathrm{Zn}\left(\mathrm{NO}_{3}\right)_{2} 6 \mathrm{H}_{2} \mathrm{O}\right]$; SNW-1 = redes de base Schiff; MIL-101 $=\mathrm{Cr}_{3}(\mathrm{OH})\left(\mathrm{H}_{2} \mathrm{O}\right)_{2} \mathrm{O}\left[\left(\mathrm{O}_{2} \mathrm{C}\right) \mathrm{C}_{6} \mathrm{H}_{4}\left(\mathrm{CO}_{2}\right)\right]_{3} \cdot \mathrm{nH}_{2} \mathrm{O} ; \mathrm{BTC}=1,3,5$-benzeno tricarboxilato; MIL-68 $=\mathrm{Al}(\mathrm{OH})\left(\mathrm{O}_{2} \mathrm{C}_{-} \mathrm{C}_{6} \mathrm{H}_{4}\right.$ - $\left.\mathrm{CO}_{2}\right)$; $\left(\mathrm{MeIm}^{2}\right.$ metilimidazol); $\mathrm{THC}=$ tetra-hidrocanabinol.

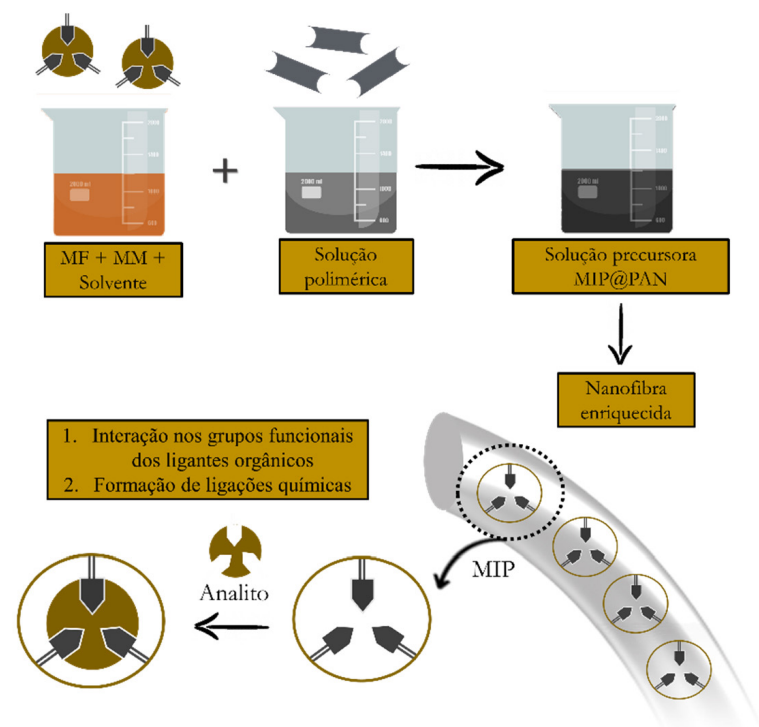

Figura 7. Ilustração esquemática da obtenção de nanofibras enriquecidas com MIP via Eletrofiação e das interações envolvidas na sorção de analitos

interações fortes entre o polímero e a molécula molde, garantindo uma distribuição homogênea dos locais de ligação nas nanofibras eletrofiadas. ${ }^{67-69} \mathrm{~A}$ Tabela 2 apresenta os principais trabalhos relatados nos últimos anos, visando o desenvolvimento e utilização de nanofibras poliméricas incorporadas com MIPs para aplicações em preparo de amostras/adsorção.
Diversos MIPs eletrofiados foram relatados na literatura nos últimos anos, utilizando de diversos polímeros precursores incorporados à diferentes monômeros funcionais para síntese de MIP. Em um trabalho de Liu e col. foram produzidas membranas de nanofibras eletromagnéticas contendo MIP para determinação de Rodamina $\mathrm{B}(\mathrm{RhB})$ em amostras de água. Foi demonstrada a eficiência do material frente a algumas variáveis do preparo de amostra, como efeito do $\mathrm{pH}$, tempo de adsorção e quantidade de material, apresentando recuperação na faixa de $97,8 \%-117,1 \%{ }^{70}$

Já Pirdadeh-Beiranvand e col. relataram a produção de um nanocompósito de nanofibra magnética molecularmente impressa (MMIN), usada na técnica de SPE visando a extração de nilotinibe em soro humano. Vários parâmetros de influência na extração foram otimizados como pH, quantidade de material, tempo de extração, volume da amostra, reutilização e estabilidade do adsorvente, sendo, por fim, proposto um método simples e rápido, com recuperação do analito superior a $90 \% .^{72}$

Xue e col. relataram a eletrofiação de nanofibras de MIP para determinação de 2,4-dinitrotolueno em água. Após síntese e caracterização, o material foi aplicado em um estudo de adsorção e seletividade, apresentando notável estabilidade e reutilização após diversos ciclos de utilização, perdendo apenas 3\% de seu desempenho após oito ciclos. Os autores sugeriram o uso do material desenvolvido para futuro rastreamento do explosivo. ${ }^{73}$

Visando a determinação de íons $\mathrm{Cu}(\mathrm{II}), \mathrm{Cd}(\mathrm{II}), \mathrm{Pb}(\mathrm{II})$ e $\mathrm{Ni}(\mathrm{II})$, Li e col., Rammika e col. e Liu e col. desenvolveram materiais adsorventes aplicados em solução aquosa e águas residuais. ${ }^{82,83,85} \mathrm{Li}$ e col. propuseram a síntese de nanofibras de quitosana eletrofiadas 
Tabela 2. Nanofibras de MIPs reportadas na literatura para aplicações em adsorção e/ou preparo de amostras em diferentes matrizes

\begin{tabular}{|c|c|c|c|c|}
\hline Analito & Material & Amostra & Condições da eletrofiação & Ref. \\
\hline Rodamina-B & PET@MIP & Solução aquosa & $\begin{array}{c}\text { Tensão: } 18 \mathrm{KV} \\
\text { Distância do coletor: } 15 \mathrm{~cm} \\
\text { Tipo de coletor: cilíndrico coberto por papel } \\
\text { alumínio } \\
\mathrm{T}=25^{\circ} \mathrm{C} \text { umidade }=45 \%\end{array}$ & 70 \\
\hline Bisfenol-A & PES@MIP & Solução aquosa & $\begin{array}{c}\text { Vazão: } 6 \mathrm{~mL} \mathrm{~h}^{-1} \\
\text { Tensão: } 15 \mathrm{KV} \\
\text { Distância do coletor: } 6 \mathrm{~cm} \\
\text { Tipo de coletor: água destilada } \\
\text { Temperatura e umidade ambiente } \\
\end{array}$ & 71 \\
\hline Nilobitine & PAN@MIP & Soro humano & $\begin{array}{c}\text { Vazão: } 1 \mathrm{~mL} \mathrm{~h}^{-1} \\
\text { Tensão: } 17,5 \mathrm{KV} \\
\text { Distância do coletor: } 19 \mathrm{~cm} \\
\text { Temperatura e umidade ambiente }\end{array}$ & 72 \\
\hline Herbicida (2,4-D) & PET@MIP & Solução aquosa & $\begin{array}{c}\text { Tensão: } 30 \mathrm{KV} \\
\text { Distância do coletor: } 20 \mathrm{~cm} \\
\text { Tipo de coletor: folha de papel alumínio } \\
\text { Temperatura e umidade ambiente }\end{array}$ & 73 \\
\hline Estrona & PMA@MIP & Solução padrão & $\begin{array}{c}\text { Vazão: } 0,2 \mathrm{~mL} \mathrm{~h}^{-1} \\
\text { Tensão: } 20 \mathrm{KV} \\
\text { Distância do coletor: } 15 \mathrm{~cm} \\
\text { Tipo de coletor folha de papel alumínio } \\
\text { Temperatura e umidade ambiente } \\
\end{array}$ & 74 \\
\hline DNT & PAM@MIP & Solução aquosa & $\begin{array}{c}\text { Vazão: } 0,1 \mathrm{~mm} \mathrm{~min}^{-1} \\
\text { Tensão: } 10-12 \mathrm{KV} \\
\text { Distância do coletor: } 10 \mathrm{~cm} \\
\text { Tipo de coletor: folha de papel alumínio } \\
\text { Temperatura e umidade ambiente } \\
\end{array}$ & 75 \\
\hline $\begin{array}{l}\text { Atrazina desisopropil, atraton, } \\
\text { carboxina, linuron e clorpirifós }\end{array}$ & PS@MIP & $\begin{array}{c}\text { Solução aquosa e águas } \\
\text { de rios }\end{array}$ & $\begin{array}{c}\text { Vazão: } 10 \mu \mathrm{L} \mathrm{min}^{-1} \\
\text { Tensão: } 10 \mathrm{KV} \\
\text { Distância do coletor: } 15 \mathrm{~cm} \\
\text { Tipo de coletor: folha de papel alumínio } \\
\text { Temperatura ambiente e umidade }=40 \%\end{array}$ & 76 \\
\hline Fenilalanina & Quitosana@MIP & Solução padrão & $\begin{array}{c}\text { Vazão: } 30 \mathrm{~mm}^{3} \mathrm{~min}^{-1} \\
\text { Tensão: } 30 \mathrm{KV} \\
\text { Distância do coletor: } 10 \mathrm{~cm} \\
\text { Tipo de coletor: folha de papel alumínio } \\
\mathrm{T}=26^{\circ} \mathrm{C} \\
\end{array}$ & 77 \\
\hline Compostos organossulfura-dos & Quitosana@MIP & Combustível & $\begin{array}{c}\text { Vazão: } 1,2 \mathrm{~mL} \mathrm{~h}^{-1} \\
\text { Tensão: } 30 \mathrm{KV} \\
\text { Distância do coletor: } 17 \mathrm{~cm} \\
\text { Tipo de coletor: folha de papel alumínio }\end{array}$ & 78 \\
\hline Azul de metileno & PVA@MIP & Águas residuais & $\begin{array}{c}\text { Tensão: } 15 \mathrm{KV} \\
\text { Distância do coletor: } 18 \mathrm{~cm} \\
\text { Tipo de coletor: folha de papel alumínio }\end{array}$ & 79 \\
\hline Acessulfame & TMSPA@MIP & Bebidas & $\begin{array}{c}\text { Tensão: } 15 \mathrm{KV} \\
\text { Distância do coletor: } 15 \mathrm{~cm} \\
\text { Tipo de coletor: folha de papel alumínio } \\
\text { Temperatura ambiente }\end{array}$ & 80 \\
\hline Acessulfame & TMSPA@MIP & $\begin{array}{l}\text { Bebidas na presença de } \\
\text { doces selecionados }\end{array}$ & $\begin{array}{c}\text { Tensão: } 15 \mathrm{KV} \\
\text { Distância do coletor: } 15 \mathrm{~cm} \\
\text { Tipo de coletor: barra de aço inoxidável } \\
\text { Temperatura ambiente } \\
\end{array}$ & 81 \\
\hline Íons $\mathrm{Cd}$ (II) e $\mathrm{Pb}$ (II) & Quitosana@MIP & Solução aquosa & $\begin{array}{c}\text { Vazão: } 0,6 \mathrm{~mL} \mathrm{~h}^{-1} \\
\text { Tensão: } 19 \mathrm{KV} \\
\text { Distância do coletor: } 9,5 \mathrm{~cm} \\
\text { Tipo de coletor: folha de papel alumínio }\end{array}$ & 82 \\
\hline Íon Ni(II) & PSF@MIP & $\begin{array}{l}\text { Água do mar e rio, esgoto } \\
\text { não tratado e água tratada }\end{array}$ & $\begin{array}{c}\text { Vazão: } 0,45 \mathrm{~mL} \mathrm{~h}^{-1} \\
\text { Tensão: } 15 \mathrm{KV} \\
\text { Distância do coletor: } 12 \mathrm{~cm} \\
\text { Tipo de coletor: folha de papel alumínio }\end{array}$ & 83 \\
\hline Íon Th(IV) & Quitosana@MIP & Efluentes & $\begin{array}{c}\text { Vazão: } 0,4 \mathrm{~mL} \mathrm{~h}^{-1} \\
\text { Tensão: } 15 \mathrm{KV} \\
\text { Distância do coletor: } 15 \mathrm{~cm} \\
\text { Tipo de coletor: placa metálica } \\
\text { Temperatura: } 40^{\circ} \mathrm{C}\end{array}$ & 84 \\
\hline
\end{tabular}


Tabela 2. Nanofibras de MIPs reportadas na literatura para aplicações em adsorção e/ou preparo de amostras em diferentes matrizes (cont.)

\begin{tabular}{|c|c|c|c|c|}
\hline Analito & Material & Amostra & Condições da eletrofiação & Ref \\
\hline Íon $\mathrm{Cu}$ (II) & PVA@MIP & Solução aquosa & $\begin{array}{c}\text { Vazão: } 0,4 \mathrm{~mL} \mathrm{~h}^{-1} \\
\text { Tensão: } 26 \mathrm{KV} \\
\text { Distância do coletor: } 7 \mathrm{~cm} \\
\text { Tipo de coletor: folha de alumínio }\end{array}$ & 85 \\
\hline Derivados de parabenos & PS@MIP & $\begin{array}{l}\text { Água engarrafada, água de } \\
\text { torneira e água do mar }\end{array}$ & $\begin{array}{c}\text { Vazão: } 1,0 \mathrm{~mL} \mathrm{~h}^{-1} \\
\text { Tensão: } 30 \mathrm{KV} \\
\text { Distância do coletor: } 15 \mathrm{~cm} \\
\text { Tipo de coletor: folha de alumínio }\end{array}$ & 86 \\
\hline Clorpirifós & PVA@MIP & $\begin{array}{c}\text { Água de poço e esgoto } \\
\text { agrícola }\end{array}$ & $\begin{array}{c}\text { Vazão: } 0,5 \mathrm{~mL} \mathrm{~h}^{-1} \\
\text { Tensão: } 20 \mathrm{KV} \\
\text { Distância do coletor: } 10 \mathrm{~cm}\end{array}$ & 87 \\
\hline Cinchonidina & PAN@MIP & $\begin{array}{c}\text { Água de poço e esgoto } \\
\text { agrícola }\end{array}$ & $\begin{array}{c}\text { Vazão: } 8 \mu \mathrm{min}^{-1} \\
\text { Tensão: } 15 \mathrm{KV} \\
\text { Distância do coletor: } 10 \mathrm{~cm} \\
\text { Tipo de coletor: folha de alumínio } \\
\text { Temperatura e umidade ambiente }\end{array}$ & 88 \\
\hline
\end{tabular}

Bilirrubina $\quad$ PES@MIP Sangue

Tensão: $18 \mathrm{KV}$

Distância do coletor: $15 \mathrm{~cm}$

Tipo de coletor: folha de alumínio

Temperatura: $25^{\circ} \mathrm{C}$ e umidade: $43-47 \%$

$$
\text { Tensão: } 15,7 \mathrm{KV}
$$

Distância do coletor: $138 \mathrm{~mm}$

Tipo de coletor: folha de alumínio

Temperatura ambiente

$$
\text { Tensão: } 17 \mathrm{KV}
$$

Distância do coletor: $15 \mathrm{~cm}$

Tipo de coletor: tambor rotativo $700 \mathrm{rpm}$

Temperatura ambiente

Tensão: 12-15 KV

Distância do coletor: $13 \mathrm{~cm}$

Tipo de coletor: placa metálica

Temperatura ambiente

Vazão: $0,55 \mathrm{~mL} \mathrm{~h}^{-1}$

Tensão: $25 \mathrm{KV}$

Distância do coletor: $13,9 \mathrm{~cm}$

Tipo de coletor: cilindro coberto por papel alumínio

Temperatura ambiente

Vazão: $0,3 \mathrm{~mL} \mathrm{~h}^{-1}$

Tensão: $20 \mathrm{KV}$

Distância do coletor: $15 \mathrm{~cm}$

Tipo de coletor: placa metálica de alumínio

Vazão: 0,07 $\mathrm{mL} \mathrm{h}^{-1}$

Tensão: $16 \mathrm{KV}$

Distância do coletor: $15 \mathrm{~cm}$

Tipo de coletor: folha de alumínio

Temperatura ambiente

Umidade: $40 \%$

\begin{tabular}{|c|c|}
\hline Propanolol & PET@MIP \\
\hline
\end{tabular}

Tensão: $20 \mathrm{KV}$

Tipo de coletor: folha de papel alumínio

90 
técnicas de SPE ainda se mostra pouco realizada devido à possível redução da permeabilidade da fase sólida, ocasionada por eventuais formações de aglomerados na solução da amostra..$^{97-99}$

Nesse sentido, o enriquecimento nanofibras com NPs tem sido uma estratégia eficiente e frequentemente relatada na literatura, afim de melhorar as propriedades mecânicas e estruturais, além da capacidade de interações químicas e físicas das NPs (Figura 8). No que se refere as principais limitações do uso de NPs incorporadas às nanofibras, sabe-se que altas porcentagens de NPs podem impedir o processo de eletrofiação, enquanto baixas porcentagens podem não atribuir o efeito requerido. A Tabela 3 apresenta os principais trabalhos relatados nos últimos anos, visando o desenvolvimento e utilização de nanofibras poliméricas incorporadas com NPs para aplicações em preparo de amostras/adsorção.
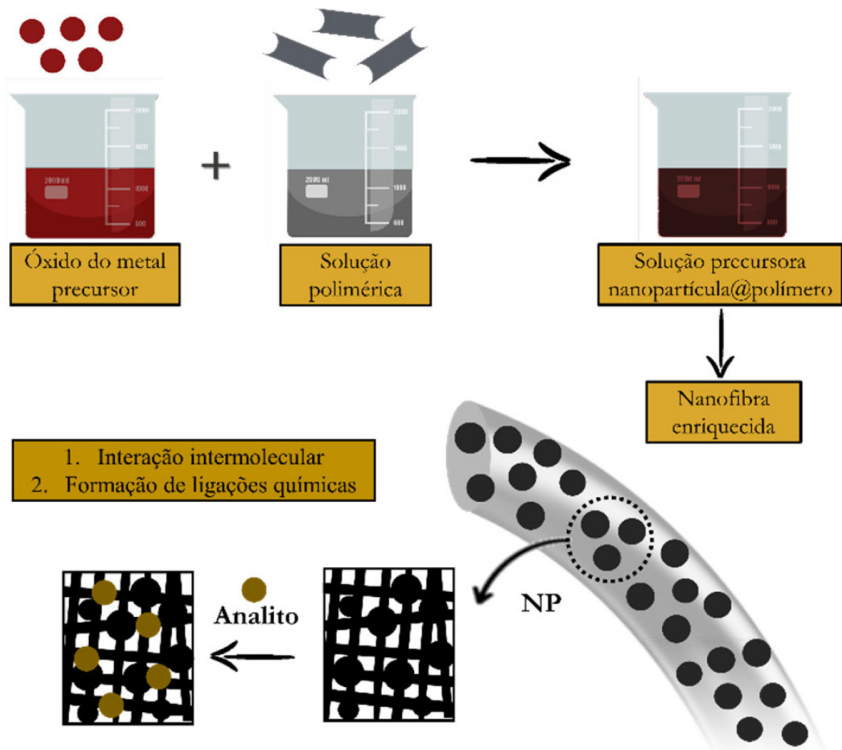

Figura 8. Ilustração esquemática da obtenção de nanofibras enriquecidas com NP via eletrofiação e das interações envolvidas na sorção de analitos

Dentre inúmeras nanofibras enriquecidas com NPs que foram descritas na literatura para diversas aplicações, pode-se destacar nanofibras aplicadas para a determinação de íons $\mathrm{Pb}(\mathrm{II}), \mathrm{Cd}(\mathrm{II})$, $\mathrm{Cr}(\mathrm{VI}), \mathrm{Cu}(\mathrm{II}), \mathrm{Ln}(\mathrm{III}), \mathrm{As}(\mathrm{IV})$ e Zn(II). Trabalhos como o de Hota e col., Wang e col. e Shariful e col. descreveram o desenvolvimento de materiais poliméricos enriquecidos com NPs para extração dos íons em solução padrão. ${ }^{101-103}$ Em 2018, Haddad e Alharbi relataram um aumento nas capacidades de adsorção dos íons $\mathrm{Pb}$ (II) e $\mathrm{Cd}$ (II) de soluções aquosas utilizando nanofibras de poliacrilonitrila enriquecidas com $\mathrm{ZnO} .{ }^{104}$ No mesmo ano, Li e col. relataram o desenvolvimento de fibras compostas por óxido de polietileno/ quitosana, enriquecidas com nanotubos de haloisita e óxido de ferro $\left.\left(\mathrm{Al}_{2} \mathrm{Si}_{2} \mathrm{O}_{5}(\mathrm{OH})_{4}\right) / \mathrm{Fe}_{3} \mathrm{O}_{4}\right)$, cujos materiais apresentaram grande área de superfície específica e propriedades superparamagnéticas com alta eficiência para extração de metais pesados em água. ${ }^{105}$

Recentemente, Alharbi e col. desenvolveram membranas de nanofibras funcionalizadas com óxidos metálicos para adsorção de $\mathrm{Pb}$ (II) e Cd(II). Em estudos de adsorção, os autores demonstraram após a otimização parâmetros como pH da solução, concentrações iniciais de íons e tempo de interação, recuperações próximas de $100 \%$ para ambos os íons. ${ }^{106}$ Trabalhando com amostras gasosas, Bajaj e col. desenvolveram nanofibras de carbono ativado enriquecido com NPs de óxido de cobre para adsorção de sulfeto de hidrogênio. O material foi sintetizado, caracterizado e aplicado em testes de adsorção. Os autores relataram que o material funcionalizado exibiu um aumento significativo na capacidade de adsorção quando comparado com o suporte polimérico puro. ${ }^{107}$

Para purificação de águas residuais, Chen e col. estudaram nanofibras de polivinilpirrolidona enriquecidas com $\mathrm{ZnO} / \mathrm{SnO}_{2}$, aplicadas para fotodegradação do corante vermelho do congo. Os autores relataram que capacidade de fotodegradação do corante pelo material pode ser atribuída à alta capacidade de adsorção, proveniente da forte interação eletrostática entre as NPs incorporadas e o corante vermelho do Congo. ${ }^{108}$ Nesse mesmo sentido, Gao e col., Pant e col., Shen e col. e Zhou e col. sintetizaram nanofibras visando a adsorção de corantes em água. Gao e col. produziram nanofibras de PVA incorporadas com NPs de $\alpha-\mathrm{Fe}_{2} \mathrm{O}_{3}$, com eficiente adsorção de laranja de metila, Pant e col. relataram o estudo de nanofibras de PVP incorporadas com NPs de $\mathrm{CdS} / \mathrm{TiO}_{2}$ suportadas em nanofibras de carbono para alta eficiência de adsorção e decomposição simultânea de azul de metileno, preto reativo 5 e laranja reativo $16 .^{109,110}$

Já Shen e col. trabalharam com nanofibras de PVP enriquecidas com alumina mesoporosa para aplicação na adsorção do corante laranja de metila. O material apresentou um bom desempenho na adsorção do corante, além de estrutura altamente estável e boa reutilização. ${ }^{111}$ Zhou e col. desenvolveram membrana nanofibrosas de PVA revestida com $\mathrm{TiO}_{2}$ para separação de óleo/água e adsorção de azul de metileno. Os autores obtiveram alto fluxo de permeação e boa eficiência de filtração ideal. ${ }^{112}$

Demirci e col. tiveram por objetivo a adsorção de DNA através da modificação de nanofibras de acetato de celulose. Após sintetizado e caracterizado, foram feitos estudos de adsorção e reutilização do material, cujos resultados sugeriram o potencial das nanofibras para aplicações biotecnológicas. ${ }^{118}$ Bagheri e col. também sintetizaram nanocompósito eletrofiados incorporados com $\mathrm{Fe}_{3} \mathrm{O}_{4}$ para extração sob campo magnético de furosemida, naproxeno, diclofenaco e propionato de clobetasol. O método foi validado visando a determinação dos fármacos selecionados em amostras de urina e os resultados indicaram que este método tem potencial suficiente para enriquecimento e determinação dos analitos na amostra de urina obtendo recuperação na faixa de $78-91 \%$. $^{122}$

\section{NANOFIBRAS ENRIQUECIDAS VS MIP/MOF/NP ISOLADOS: COMPARAÇÃO DE DESEMPENHO E CONFIGURAÇÕES DE USO}

Conforme já descrito, a eletrofiação permite produzir fibras unidimensionais (ex.: fios únicos), bidimensionais (ex.: folhas e filmes finos) e tridimensionais (ex.: nanoestruturas), atribuindo inúmeras possibilidades de configurações de fibras únicas ou arranjos de múltiplas fibras, em diversos formatos e configurações existentes de SPE e SPME, embora diferentes disposições das fibras levem a efeitos significativos no desempenho dos dispositivos analíticos. ${ }^{1,38}$ Notadamente, a maior parte dos trabalhos que tratam da utilização de nanofibras em dispositivos SPE/SPME, reportam aplicações de múltiplas fibras nos formatos de discos, ${ }^{123,124}$ membranas ${ }^{125}$ e microcolunas ${ }^{126,127}$ no modo off-line. Contudo, limitações relacionadas a reprodutibilidade do preparo desses dispositivos têm sido relatadas na literatura.

Em contrapartida, alguns autores sugerem que utilização de peças únicas na extração direta por SPME, seja por imersão direta da fibra na matriz ou por headspace, facilitam a difusão do analito para a fibra e propicia melhores recuperações e reprodutibilidade, ${ }^{128,129}$ embora essa estratégia seja menos recorrente. Alternativamente, o desenvolvimento dos materiais enriquecidos também é apontado como uma estratégia eficiente, afim de reduzir os problemas relativos ao empacotamento e melhorar o desempenho analítico. ${ }^{1,38} \mathrm{~A}$ Tabela 4 apresenta parâmetros relacionados às técnicas de SPE/SPME e testes 
Tabela 3. Nanofibras de NPs reportadas na literatura para aplicações em adsorção e/ou preparo de amostras em diferentes matrizes

\begin{tabular}{|c|c|c|c|c|}
\hline Analito & Material & Amostra & Condições da eletrofiação & Ref. \\
\hline BSA & HAp@CTA & Solução padrão & $\begin{array}{c}\text { Tensão: } 20-25 \mathrm{KV} \\
\text { Distância do coletor: } 12-16 \mathrm{~cm} \\
\text { Tipo de coletor: eletrodo de alumínio } \\
\mathrm{T}=25^{\circ} \mathrm{C} \text { umidade }=55 \%\end{array}$ & 100 \\
\hline Íon Cd(II) & AlOOH@PCL & Solução padrão & $\begin{array}{c}\text { Vazão: } 1 \mathrm{~mL} \mathrm{~h}^{-1} \\
\text { Tensão: } 10-20 \mathrm{KV} \\
\text { Tipo de coletor: tambor rotativo }\end{array}$ & 101 \\
\hline Íon Ln(III) & $\mathrm{SiO}_{2} @ \mathrm{PAA}$ & Solução padrão & $\begin{array}{c}\text { Vazão: } 20 \mu \mathrm{L} \mathrm{min}^{-1} \\
\text { Tensão: } 24 \mathrm{KV} \\
\text { Distância do coletor: } 15 \mathrm{~cm} \\
\text { Tipo de coletor: rolo metálico giratório }\end{array}$ & 102 \\
\hline
\end{tabular}

Temperatura $=25 \pm 2{ }^{\circ} \mathrm{C}$ e umidade $=42 \pm 5 \%$

\begin{tabular}{|c|c|c|c|}
\hline Íons $\mathrm{Cu}(\mathrm{II}), \mathrm{Zn}$ (II) e $\mathrm{Pb}$ (II) & PEO@quitosana & Solução padrão & $\begin{array}{c}\text { Vazão: 0,1-0,5 mL h } \\
\text { Tensão: 7-15 KV } \\
\text { Tensão: } 25 \mathrm{KV}\end{array}$ \\
\hline
\end{tabular}

Tipo de coletor: aço inoxidável

\section{Vazão: $1,5 \mathrm{~mL} \mathrm{~h}^{-1}$}

Tensão: $20 \mathrm{KV}$

Íons $\mathrm{Pb}(\mathrm{II})$ e $\mathrm{Cd}(\mathrm{II})$

ZnO@PAN Solução aquosa

Distância do coletor: $18 \mathrm{~cm}$

Tipo de coletor: disco giratório circular

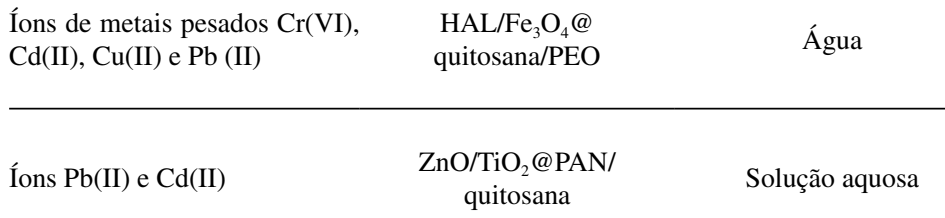
Tensão: $22 \mathrm{KV}$

Distância do coletor: $10 \mathrm{~cm}$

Temperatura ambiente Umidade $<10 \%$

Vazão: $0,2 \mathrm{~mL} \mathrm{~h}^{-1}$ Tensão: $25 \mathrm{KV}$

Distância do coletor: $10 \mathrm{~cm}$

Temperatura ambiente Vazão: $0.8 \mathrm{~mL} \mathrm{~h}^{-1}$

$$
\text { Tensão: } 15 \mathrm{KV}
$$

Distância do coletor: $16 \mathrm{~cm}$

Sulfeto de hidrogênio

$\mathrm{Cu}(\mathrm{OAc})_{2} @ \mathrm{PAN}$

Gás

Tipo de coletor: coletor metálico coberto por

folha de papel alumínio

\begin{tabular}{lll}
\hline Vermelho do congo & $\mathrm{ZnO} / \mathrm{SnO}_{2} @ \mathrm{PVP}$ & Águas residuais \\
\hline
\end{tabular}

Corante laranja de metila

$\alpha-\mathrm{Fe}_{2} \mathrm{O}_{3} @ \mathrm{PVA}$

Água Tensão: $15 \mathrm{KV}$

Tipo de coletor: folha de papel alumínio

Vazão: $1 \mathrm{~mL} \mathrm{~h}^{-1}$

Tensão: $12 \mathrm{KV}$

Distância do coletor: $14 \mathrm{~cm}$

Tipo de coletor: rotativo de aço inoxidável (150

109 rpm)

Temperatura ambiente Tensão: $20 \mathrm{KV}$

Azul de metileno, preto reativo-5 e laranja reativo-16 $\mathrm{CdS} / \mathrm{TiO}_{2} @$
$\mathrm{PVP}$

Solução padrão

Distância do coletor: $15 \mathrm{~cm}$

Tipo de coletor: folha de alumínio

Temperatura ambiente

Vazão: $0,8 \mathrm{~mL} \mathrm{~h}^{-1}$ Tensão: $18 \mathrm{KV}$

Solução aquosa

tor: folha de papel alumínio

Tipo de coletor: folha de pape
Vazão: $1,0 \mathrm{~mL} \mathrm{~h}^{-1}$
Tensão: $18 \mathrm{KV}$

Tipo de coletor: folha de pape
Vazão: $1,0 \mathrm{~mL} \mathrm{~h}^{-1}$
Tensão: $18 \mathrm{KV}$

Distância do coletor: $17 \mathrm{~cm}$

Azul de metileno

$\mathrm{TiO}_{2} @ \mathrm{PLA}$

Solução aquosa

Tipo de coletor: placa metálica coberta com folha

de papel alumínio

Temperatura $=23 \pm 5{ }^{\circ} \mathrm{C}$ e umidade $=41 \pm 5 \%$

Contaminantes orgânicos
(corantes e fenol)

Tetraciclina

$\mathrm{Fe}_{3} \mathrm{O}_{4} @ \mathrm{PAN}$

Solução aquosa

Águas residuais

$\mathrm{FeCl}_{3} @ \mathrm{PAN}$

Aguas residuais

Tetraciclina

Íon Arsênio(IV)

$\mathrm{Fe}\left(\mathrm{NO}_{3}\right)_{3} \cdot 9 \mathrm{H}_{2} \mathrm{O} @ \quad$ Água subterrânea contami-
PAN/PEI

Temperatura $=23 \pm 5{ }^{\circ} \mathrm{C}$

Vazão: $0,9 \mathrm{~mL} \mathrm{~h}^{-1}$
Tensão: $12 \mathrm{KV}$

Distância do coletor: $15 \mathrm{~cm}$

Tipo de coletor: tambor metálico giratório

coberto por folha de papel alumínio
Vazão: $0,5 \mathrm{~mL} \mathrm{~h}^{-1}$

Tensão: $10 \mathrm{KV}$

Distância do coletor: $15 \mathrm{~cm}$

Coletor: alumínio

Temperatura ambiente 
Tabela 3. Nanofibras de NPs reportadas na literatura para aplicações em adsorção e/ou preparo de amostras em diferentes matrizes (cont.)

\begin{tabular}{|c|c|c|c|c|}
\hline Analito & Material & Amostra & Condições da eletrofiação & Ref. \\
\hline Íon $\mathrm{Pb}(\mathrm{II})$ & FumF@PAN & Solução aquosa & $\begin{array}{c}\text { Vazão: } 1 \mathrm{~mL} \mathrm{~h}^{-1} \\
\text { Tensão: } 22 \mathrm{KV} \\
\text { Distância do coletor: } 9 \mathrm{~cm} \\
\text { Temperatura: } 23^{\circ} \mathrm{C} \text { e umidade }=20 \%\end{array}$ & 116 \\
\hline Vermelho do congo & $\mathrm{ZnO} / \mathrm{SnO}_{2} @ \mathrm{PVP}$ & Águas residuais & $\begin{array}{c}\text { Tensão: } 15 \mathrm{KV} \\
\text { Tipo de coletor: folha de papel alumínio }\end{array}$ & 117 \\
\hline DNA & CPAD@CA & Solução aquosa & $\begin{array}{c}\text { Vazão: } 1 \mathrm{~mL} \mathrm{~h}^{-1} \\
\text { Tensão: } 15 \mathrm{KV} \\
\text { Distância do coletor: } 10 \mathrm{~cm} \\
\text { Tipo de coletor: folha de papel alumínio } \\
\text { Temperatura: } 25^{\circ} \mathrm{C} \\
\text { Umidade }=25 \% \\
\end{array}$ & 118 \\
\hline Malaquita e leucomalaquita & GO/TiO $2 @ \mathrm{PAN}-\mathrm{CMA}$ & Solução aquosa & $\begin{array}{c}\text { Vazão: } 0,020 \mathrm{~mL} \mathrm{~min}^{-1} \\
\text { Tensão: } 14 \mathrm{KV} \\
\text { Distância do coletor: } 10 \mathrm{~cm} \\
\text { Tipo de coletor: folha de papel alumínio } \\
\text { Temperatura: } 40 \pm 2{ }^{\circ} \mathrm{C} \\
\text { Umidade }=25 \pm 1,2 \%\end{array}$ & 119 \\
\hline Íon $\mathrm{Cu}$ (II) & Si-MO@PVA/PAA & Solução aquosa & $\begin{array}{c}\text { Vazão: } 0,5 \mathrm{~mL} \mathrm{~h}^{-1} \\
\text { Tensão: } 15 \mathrm{KV} \\
\text { Distância do coletor: } 20 \mathrm{~cm}\end{array}$ & 120 \\
\hline Compostos orgânicos voláteis & NiO@PBT & Solução aquosa & $\begin{array}{c}\text { Vazão: } 0,5 \mathrm{~mL} \mathrm{~h}^{-1} \\
\text { Tensão: } 16 \mathrm{KV} \\
\text { Distância do coletor: } 10 \mathrm{~cm}\end{array}$ & 121 \\
\hline $\begin{array}{l}\text { Furosemida, naproxeno, diclof- } \\
\text { enaco e propionato de clobetasol }\end{array}$ & $\mathrm{Fe}_{3} \mathrm{O}_{4} @ \mathrm{PBT}$ & Urina & $\begin{array}{c}\text { Vazão: } 0,15 \mathrm{~mL} \mathrm{~min}^{-1} \\
\text { Tensão: } 16 \mathrm{KV} \\
\text { Distância do coletor: } 10 \mathrm{~cm} \\
\text { Tipo de coletor: folha de papel alumínio }\end{array}$ & 122 \\
\hline
\end{tabular}

Hap $=\left(\mathrm{Ca}_{10}\left(\mathrm{PO}_{4}\right)_{6}(\mathrm{OH})_{2}\right) ; \mathrm{CTA}=$ triacetato de celulose $; \mathrm{PAN}=$ poliacrilonitrila $; \mathrm{Fe}(\mathrm{acac})_{3}=$ acetilacetonato de ferro; PVA = poli $($ álcool vinílico $) ; \mathrm{PVP}=$ polivinilpirrolidona OAc $=$ Acetato $; \mathrm{HAL}=$ nanotubos de haloisita; $\mathrm{PEO}=$ óxido de polietileno; $\mathrm{PCL}=$ policaprolactona; $\mathrm{PAA}=$ poli $($ ácido acrílico) PEI = polietilenimina; FumF = fumarato de ferro (II); PES = polietersulfona; PLA = metiltriclorossilano; CA= acetato de celulose; CPAD = ácido 4-cianopentanóico ditiobenzoato; $\mathrm{GO}=$ óxido de grafeno; $\mathrm{PAN}-\mathrm{CMA}=$ poli (acrilonitrila-co-ácido maleico) $\mathrm{PA}=$ poliamida; $\mathrm{PBT}=$ tereftalato de polibutileno; $\mathrm{PT}=$ politiofeno; $\mathrm{Si}-\mathrm{MO}=$ sílica modificada; $\mathrm{BSA}=$ albumina sérica bovina.

de adsorção com uso de adsorventes MOFs, MIPs e NPs incorporados a matrizes eletrofiadas e isolados, como forma de comparação do desempenho desses materiais.

Em 2018, Yan e col. destacaram que nanofibras são suportes ideais para as MOFs, por propiciarem a formação de redes em 3D, evitando a agregação das partículas, melhorando a dispersão e disponibilizando mais sítios ativos para adsorção. Além disso, os autores destacaram serem necessários menores volumes de solventes de eluição e menor quantidade de material sorvente na extração. ${ }^{136}$ Recentemente, Wang e col. destacaram que a eletrofiação de materiais enriquecidos com MOFs, comparada com outros métodos de síntese, requer menor consumo de reagentes, demonstrando a vantagem ecológica da técnica. ${ }^{137}$ De acordo com os dados apresentados na Tabela 4, verificase que os materiais eletrofiados a base de PAN e PS enriquecidos com

Tabela 4. Parâmetros relacionados às técnicas de SPE/SPME e testes de adsorção com uso de adsorventes MOFs, MIPs e NPs isolados ou incorporados a matrizes eletrofiadas

\begin{tabular}{|c|c|c|c|c|c|c|c|c|c|c|}
\hline & Material sorvente & $\begin{array}{c}\text { Área de } \\
\text { superfície } \\
\left(\mathrm{m}^{2} \mathrm{~g}^{-1}\right)\end{array}$ & $\begin{array}{l}\text { Capacidade de } \\
\text { adsorção (mg } \\
\left.\mathrm{g}^{-1}\right)\end{array}$ & Técnica & $\begin{array}{l}\text { Quantidade } \\
\text { de sorvente }\end{array}$ & Reuso & $\begin{array}{l}\text { Volume de } \\
\text { amostra }\end{array}$ & $\begin{array}{c}\text { Volume de } \\
\text { solvente } \\
\text { eluição }\end{array}$ & Modo & Ref. \\
\hline \multirow{4}{*}{ MOFs } & MIL-68(Al)@PAN & 1460 & 306 & SPE & $6,0 \mathrm{mg}$ & 10 ciclos & $80 \mathrm{~mL}$ & $4,0 \mathrm{~mL}$ & Off-line & 51 \\
\hline & MIL-68(Al) & 175 & 11,55 & PT-SPE & $8,0 \mathrm{mg}$ & 100 vezes & $2 \mathrm{~mL}$ & $200 \mu \mathrm{L}$ & Off-line & 130 \\
\hline & MOF-199@PS & 182,60 & 8,50 & TFME & $80 \mathrm{mg}$ & - & $2,0 \mathrm{~mL}$ & $240 \mu \mathrm{L}$ & Off-line & 52 \\
\hline & MOF-199 & 77,47 & 3,74 & MSPE & $20 \mathrm{mg}$ & - & $4,0 \mathrm{~mL}$ & $3,0 \mathrm{~mL}$ & Off-line & 131 \\
\hline \multirow{4}{*}{ MIPs } & PVA@MIP bisfenol-A & - & - & SPE & $20 \mathrm{mg}$ & - & $1,0 \mathrm{~mL}$ & $1,0 \mathrm{~mL}$ & Off-line & 90 \\
\hline & MIP bisfenol-A & 340,21 & - & SPE & $100 \mathrm{mg}$ & - & $5,0 \mathrm{~mL}$ & $3,0 \mathrm{~mL}$ & Off-line & 132 \\
\hline & PVA@MIP Cu (II) & 143,00 & 143,00 & - & $100 \mathrm{mg}$ & 5 ciclos & - & - & Off-line & 85 \\
\hline & MIP Cu (II) & 120,91 & 111,81 & - & $10 \mathrm{mg}$ & 10 ciclos & - & - & Off-line & 133 \\
\hline \multirow{4}{*}{ NPs } & $\mathrm{Al}_{2} \mathrm{O}_{3} @ \mathrm{PVP}$ & 417,70 & - & - & $5,0 \mathrm{mg}$ & 5 ciclos & $30 \mathrm{~mL}$ & - & Off-line & 106 \\
\hline & $\mathrm{Al}_{2} \mathrm{O}_{3}$ & - & - & $\mu$-SPE & $5,0 \mathrm{~g}$ & 6 ciclos & $150 \mathrm{~mL}$ & $3,0 \mathrm{~mL}$ & Off-line & 134 \\
\hline & $\mathrm{Fe}_{3} \mathrm{O}_{4} @ \mathrm{PAN}$ & 17,80 & - & - & $10 \mathrm{mg}$ & 5 ciclos & $20 \mathrm{~mL}$ & - & Off-line & 114 \\
\hline & $\mathrm{Fe}_{3} \mathrm{O}_{4}$ & 71 & - & SPE & $50 \mathrm{mg}$ & 6 ou mais ciclos & $1000 \mathrm{~mL}$ & $8,0 \mathrm{~mL}$ & Off-line & 135 \\
\hline
\end{tabular}

TFME = microextração de filme fino (do inglês, thin-film microextraction). 
MIL-68(Al) e MOF-199, respectivamente, apresentam maiores valores de área superficial e capacidade de adsorção que os materiais isolados. Além disso, comparando o uso das técnicas de microextração de filme fino (TFME) e SPME, confirmam-se consumos menores de volume de amostra e solvente de eluição ao se empregar materiais eletrofiados.

Patel e col. realçaram o desafio de unir as técnicas de eletrofiação e impressão molecular, utilizando de nanofibras como suportes para MIPs, produzindo materiais enriquecidos de alta estabilidade e robustez, aumentando, ainda, a limitada capacidade de adsorção e taxa de transferência de MIPs. ${ }^{138}$ Novamente, de acordo com os dados apresentados na Tabela 4, pode-se verificar que o material eletrofiado a base de PVA, enriquecido com polímero de impressão de íon $\mathrm{Cu}(\mathrm{II})$, apresenta maior área superficial e capacidade de adsorção que os materiais isolados, exigindo menor quantidade de material sorvente, volume de amostra e solvente de eluição em SPE com uso de PVA@MIP bisfenol-A, quando comparado com SPE com MIP bisfenol-A.

Já Bagheri e col. evidenciaram que a incorporação de NPs em matrizes poliméricas conduz à obtenção de melhores propriedades mecânicas e térmicas, ${ }^{139}$ além de Feizbakhsh e col. proporem que incorporação de NPs a suportes poliméricos reduz a agregação nas NPs, melhorando sua dispersão na matriz e fornecendo superfícies ativas para interagir com moléculas alvos. ${ }^{140}$ Ainda de acordo com os dados apresentados na Tabela 4, podem-se evidenciar áreas superficiais maiores para de materiais eletrofiados a base de PVP e PAN, enriquecidos com $\mathrm{Al}_{2} \mathrm{O}_{3}$ e $\mathrm{Fe}_{3} \mathrm{O}_{4}$, respectivamente, quando comparados com os óxidos puros.

\section{CONCLUSÕES}

Ao descrever nesta contribuição os fundamentos e aplicações da técnica de eletrofiação para a produção de novos materiais sorventes baseados em nanofibras poliméricas enriquecidas com MOF, MIP e NP, espera-se que tenham sido apresentadas evidências sólidas de inúmeras utilizações desses materiais em processos de adsorção e preparo de amostras com sucesso. Frente às dezenas de trabalhos relatados envolvendo a utilização desses materiais para determinação de diferentes analitos em diversos tipos de amostras (biológicas, farmacêuticas, ambientais, forenses, dentre outras), fica evidente o interesse analítico em aplicar a técnica de eletrofiação para o desenvolvimento nanofibras adsorventes com performance superior aos materiais isolados (MOF, MIP e NP) já bem relatados na literatura.

Nesse sentido, pode-se vislumbrar que a abordagem de nanofibras eletrofiadas incorporadas com MOF, MIP e NP apresenta-se como uma estratégia inovadora para obtenção de materiais porosos, de alta área superficial e boa estabilidade térmica, mecânica e química, agregada à seletividade dos MIPs, à alta eficiência de extração de moléculas de diferentes classes das MOFs, além da excelente capacidade de funcionalização das NPs. Em oposição, vale destacar também as limitações associadas à baixa reticulação e às mudanças estruturais das cavidades seletivas dos MIPs, à dessorção incompleta de analitos e baixa estabilidade químicas das MOFs, bem como à baixa permeabilidade da fase sólida de NPs.

Em particular, todos os trabalhos relatados nesta contribuição demonstram a aplicabilidade da eletrofiação para obtenção de materiais à base de soluções poliméricas

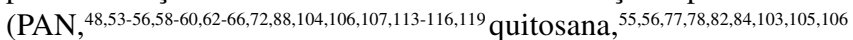
PS,${ }^{52,57,76,86}$ PET $,{ }^{70,73,92,93,96} \mathrm{PES},{ }^{71,89} \mathrm{PMA},{ }^{74} \mathrm{PAM},{ }^{75} \mathrm{PVA},{ }^{79,85,87,90,91}$ TMSPA,${ }^{80,81}$ PSF, ${ }^{83}$ PIMH,${ }^{94}$ NYLON- $6,{ }^{95}$ CTA,${ }^{100}$ PCL,${ }^{101}$ PAA, ${ }^{102,120}$ PVP, ${ }^{108,110,111,117}$ PVA, ${ }^{109}$ PLA, ${ }^{112}$ CA,${ }^{118}$ e PBT $^{121,122}$ ), sendo essa a classe mais popular de materiais aplicados em eletrofiação visando a SPE, contudo, não a única aplicável, demonstrando o ainda limitado conhecimento acerca da estrutura, obtenção e aplicabilidade das nanofibras eletrofiadas.

Nesse sentido, por fim, aponta-se a necessidade de empreender esforços na fabricação de novos aparatos experimentais de eletrofiação, novos materiais enriquecidos e novos dispositivos de (micro)extração em fase sólida, bem como de uma completa caracterização e elucidação estrutural das nanofibras eletrofiadas. Sobretudo, frente às diferentes e mais complexas matrizes analisadas nos trabalhos relatados, pode-se vislumbrar promissoras e eficientes técnicas de preparo de amostras e processos de adsorção baseados em nanofibras eletrofiadas funcionalizadas com MIP, MOF e NP e outras inúmeras combinações não abordadas neste trabalho.

\section{AGRADECIMENTOS}

Os autores agradecem à Fundação de Amparo à Pesquisa do Estado de Minas Gerais (FAPEMIG), ao Conselho Nacional de Desenvolvimento Científico e Tecnológico (CNPq) e à Coordenação de Aperfeiçoamento de Pessoal de Nível Superior - Brasil (CAPES) - Código Financeiro 001 - pelo suporte financeiro. Este trabalho também foi financiado em parte pela Rede Mineira de Química (RQ-MG) apoiado pela FAPEMIG (Projeto: REDE-113/10; Projeto: CEX-RED-0010-14).

\section{REFERÊNCIAS}

1. Chigome, S.; Torto, N.; Anal. Chim. Acta 2011, 706, 1.

2. Cui, J.; Li, F.; Wang, Y.; Zhang, Q.; Ma, W.; Huang, C.; Sep. Purif. Technol. 2020, 117116, 250.

3. Hussain, D.; Raza Naqvi, S. T.; Ashiq, M. N.; Najam-ul-Haq, M.; Talanta 2019, 120413, 208; Martins, M. A.; Trindade, T.; Quim. Nova 2012, 35, 7 .

4. Ibrahim, H. M.; Klingner, A.; Polym. Test. 2020, 106647, 90.

5. Bhardwaj, N.; Kundu, S. C.; Biotechnol. Adv. 2010, 2, 3.

6. Teo, W. E.; Ramakrishna, S.; Nanotechnology 2006, 17, 14.

7. Wang, X.; Ding, B.; Sun, G.; Wang, M.; Yu, J. Prog. Mater. Sci. 2013, 58,8 .

8. Zhang, C.-L.; Yu, S.-H.; Chem. Soc. Rev. 2014, 43, 13.

9. Ahmed, F. E.; Lalia, B. S.; Hashaikeh, R.; Desalination 2015, 356.

10. Kenry; Lim, C. T.; Prog. Polym. Sci. 2017, 70.

11. Xue, J.; Xie, J.; Liu, W.; Xia, Y.; Acc. Chem. Res. 2017, 50, 8.

12. Tucker, N.; Stanger, J. J.; Staiger, M. P.; Razzaq, H.; Hofman, K.; J. Eng. Fibers Fabr. 2012, 7, 63.

13. Xue, J.; Wu, T.; Dai, Y.; Xia, Y.; Chem. Rev. 2019, 119, 8.

14. Cooley, J. F.; US Pat. 692631 A 1902.

15. Zeleny, J.; Phys. Rev 1914, 69, 3.

16. Taylor, G.; Series A: Mathematical and Physical and Sciences 1964, 280, 1382.

17. He, J-H.; Liu, Y.; Mo, L-F.; Wan, Y.; Xu, L.; Electrospun Nanofibres and Their Applications, Smithers Rapra Update: Reino Unido, 2008.

18. Salem, D. R. Nanofibers Nanotechnology in Textiles, 2017, 3.

19. Baji, A.; Mai, Y.-W.; Wong, S.-C.; Abtahi, M.; Chen, P. Compositites Science and Technology 2010, 70, 703.

20. Rao, P. S.; Sathyanarayana, D. N.; Palaniappan, S.; Macromolecules 2002, 35, 13.

21. Yousefzadeh, M.; Latifi, M.; Teo, W.-E.; Amani-Tehran, M.; Ramakrishna, S.; Polym. Eng. Sci. 2011, 51, 323.

22. Pan, H.; Li, L.; Hu, L.; Cui, X.; Polymer. 2006, 47, 4901.

23. Khamforoush, M.; Mahjob, M.; Mater. Lett. 2011, 65, 3.

24. Wang, X.; Pan, Y.; Qin, Y.; Voigt, M.; Liu, X.; Zheng, G.; Chen, Q.; Schubert, D. W.; Liu, C.; Shen, C.; Polym. Test. 2018, 69, 478.

25. Liu, Q.; Zhu, J.; Zhang, L.; Qiu, Y.; Renewable Sustainable Energy Rev. 2018, 81,1825 . 
26. Bonadies, I.; Maglione, L.; Ambrogi, V.; Paccez, J. D.; Zerbini, L. F.; Rocha e Silva, L. F.; Picanço, N. S.; Tadei, W. P.; Grafova, I.; Grafov,A.; Carfagna, C. Eur. Polym. J. 2017, 89, 211.

27. Deitzel, J. M.; Kleinmeyer, J.; Harris, D.; Beck Tan, N. C.; Polymer 2001, 42.

28. Li, Z.; Wang, C.; Effect of Working Parameters of Electrospinning. Springer: New York, 2013.

29. Gupta, P.; Elkins, C.; Long, T. E.; Wikes, G. L.; Polymer 2005, 46, 4799.

30. Kim, S. J.; Lee, C. K.; Kim, S. I.; J. Appl. Polym. Sci. 2005, 96, 4.

31. Yarin, A.; Pourdeyhimi, B.; Ramakrishna, S.; Fundamentals and applications of micro and nanofibers: Cambridge University Press: Cambridge, 2013, cap. 5.

32. Paneva, D.; Ignatova, M.; Manolova, N.; Rashkov, I. In Nanofibers: Fabrication, Performance, and Applications; Chang, W. N., ed.; Nova Science Publishers: New York, 2009, pp 73-151.

33. Megelski, S.; Stephens, J. S.; Chase, D. B.; Rabolt, J. F.; Macromolecules 2002, 35, 8456.

34. Ki, C. S.; Baek, D. H.; Gang, K. D.; Lee, K. H.; Um, I. C.; Park, Y. H.; Polym. 2005, 46, 5094.

35. Liu, H.; Hsieh, Y. L.; J. Polym. Sci., Part B: Polym. Phys. 2002, 40, 2119.

36. Costa, R. G. F.; Polímeros 2012, 22, 2.

37. Háková, M.; Havlíková, L. C.; Švec, F.; Solich, P.; Satínský, D.; Anal. Chim. Acta 2020, 1121.

38. Chigome, S.; Torto, N.; TrAC, Trends Anal. Chem. 2012, 38, 21

39. Háková, M.; Chocholoušová Havlíková, L.; Solich, P.; Švec, F.; Šatínský, D.; TrAC, Trends Anal. Chem. 2019, 110, 81.

40. Reyes-Gallardo, E. M.; Lucena, R.; Cárdenas, S.; TrAC, Trends Anal. Chem. 2016, 84,3

41. Ahmadi, M.; Elmongy, H.; Madrakian, T.; Abdel-Rehim, M.; Anal. Chim. Acta 2017, 958, 1.

42. Blachowicz, T.; Ehrmann, A.; J. Eng. Fibers Fabr. 2020, 15, 1.

43. Chigome, S.; Darko, G.; Torto, N.; The Analyst 2011, 136, 14.

44. Noruzi, M.; J. Sci. Food Agric. 2016, 96, 14.

45. Hu, B.; He, M.; Chen, B.; Anal. Bioanal. Chem. 2015, 407, 10.

46. Li, X.; Ma, W.; Li, H.; Bai, Y.; Liu, H.; Coord. Chem. Rev. 2019, 3 97, 1 .

47. Frem, R. C. G.; Arroyos, G.; Flor, J. B. S.; Alves, R. C.; Lucena, G. N.; Silva, C. M.; Coura, M. F.; Quim. Nova 2018, 41, 10; Galaço, A. R. B. S.; Lima, J. F.; Serra, O. A.; Quím. Nova 2018, 41, 6.

48. Efome, J. E.; Rana, D.; Matsuura, T.; Lan, C. Q.; J. Mater. Chem. A 2018, 6,10 .

49. Efome, J. E.; Rana, D.; Matsuura, T.; Lan, C. Q.; ACS Appl. Mater. Interfaces 2018, 10, 22.

50. Efome, J. E.; Rana, D.; Matsuura, T.; Lan, C. Q.; Sci. Total Environ. 2019, 674, 355.

51. Asiabi, M.; Mehdinia, A.; Jabbari, A.; Microchim. Acta 2017, $184,11$.

52. Liu, F.; Xu, H.; Talanta 2017, 162, 261.

53. Asiabi, M.; Mehdinia, A.; Jabbari, A.; J. Chromatogr. A 2015, 1426, 24

54. Mohammadi, V.; Jafari, M. T.; Saraji, M.; Microchem. J. 2019, 146, 98.

55. Mei, Q.; Fu, R.; Ding, Y.; Li, L.; Wang, A.; Duan, D.; Ye, D.; J. Electroanal. Chem. 2019, 113075.

56. Zhao, R.; Tian, Y.; Li, S.; Ma, T.; Lei, H.; Zhu, G.; J. Mat. Chem. A 2019, 7, 22559.

57. Peterson, G. W.; Lu, A. X.; Epps, T. H.; ACS Appl. Mater. Interfaces 2017, 9, 37.

58. Bahrami, H.; Rezaei, B.; Jafari, M. T.; Anal. Methods 2019, 11, 1073.

59. Amini, S.; Ebrahimzadeh, H.; Seidi, S.; Jalilian, N.; Microchim Acta 2020, 187, 152.

60. Wang, J.; Cai, C.; Zhang, Z.; Li, C.; Liu,R.; Chemosphere 2020, $239,1$.

61. Asiabi, M.; Mehdinia, A.; Jabbari, A.; J. Chromatogr. A 2017, 1479, 71.

62. Arabsorkhi, B.; Sereshti, H.; Abbasi, A.; J. Sep. Sci. 2019, 42, 1500.

63. Mehrafza, N.; Saraji, M.; Sep. Sci. Plus. 2018, 1, 5.
64. Yan, Z.; Hu, B.; Li, Q.; Zhang, S.; Pang, J.; Wu, C.; J. Chromatogr. A 2019, 1584, 33.

65. Wahiduzzaman, M. R. K.; Harp, S.; Neumann, J.; Sultana, Q. N.; J. Mater. Eng. Perform. 2016, 25, 4.

66. Peng, L.; Zhang, X.; Sun, Y.; Xing, Y.; Li, C.; Environ. Res. 2020, 188, 109742.

67. Pichon, V.; Delaunay, N.; Combes, A.; Anal. Chem. 2020, 92, 16.

68. Turiel, E.; Martín-Esteban, A.; Anal. Chim. Acta 2010, 668, 2; Cassiano, N. M.; Barreiro, J. C.; Cass, Q. B.; J. Braz. Chem. Soc. 2014, 25, 1.

69. Ashley, J.; Shahbazi, M.-A.; Kant, K.; Chidambara, V. A.; Wolff, A.; Bang, D. D.; Sun, Y.; Biosens. Bioelectron. 2017, 91, 606; Tarley, C. R. T.; Sotomayor, M. D. P. T.; Kubota, L. T.; Quim. Nova 2005, 28, 6.

70. Li, L.; Liu, H.; Lei, X.; Zhai, Y.; Adv. Chem. Eng. Sci. 2012, $2,2$.

71. Zhang, Y.; Wei, Q.; Zhang, Q.; Li, J.; Yang, J.; Zhao, C.; Sep. Sci. Technol. 2011, 46, 10.

72. Pirdadeh-Beiranvand, M.; Afkhami, A.; Madrakian, T.; Anal. Bioanal. Chem. 2020, 412, 1629

73. Chronakis, I. S.; Milosevic, B.; Frenot, A.; Ye, L.; Macromolecules 2006, 39,1 .

74. Kim, W. J.; Chang, J. Y.; Mater. Lett. 2011, 65, 9.

75. Xue, X.; Lu, R.; Li, Y.; Wang, Q.; Li, J.; Wang, L.; The Analyst 2018, $143,14$.

76. Ruggieri, F.; D’Archivio, A. A.; Di Camillo, D.; Lozzi, L.; Maggi, M. A.; Mercorio, R.; Santucci, S. J.; Sep. Sci. 2015, 38, 8.

77. Yoshikawa, M.; Isezaki, J.; Sep. Technol. 2014, 3, 119.

78. Ogunlaja, A. S.; Coombes, M. J.; Torto, N.; Tshentu, Z. R.; React. Funct. Polym. 2014, 81, 61.

79. Zhao, R.; Li, X.; Sun, B.; Li, Y.; Li, Y.; Wang, C.; Chem. Res.Chin. Univ. 2017, 33, 6 .

80. Moein, M. M.; Javanbakht, M.; Karimi, M.; Akbari-Adergani, B.; J. Sep. Sci. 2015, 38, 8 .

81. Moein, M. M.; Javanbakht, M.; Karimi, M.; Akbari-Adergani, B.; Talanta 2015, 134, 340 .

82. Li, Y.; Xu, C.; Qiu, T.; Xu, X.; J. Nanosci. Nanotechnol. 2015, 15, 6.

83. Rammika, M.; Darko, G.; Torto, N.; Water SA 2011. 37, 4.

84. Gore, P. M.; Khurana, L.; Siddique, S.; Panicker, A.; Kandasubramanian, B.; Environ. Sci. Pollut. Res. 2017, 25, 4.

85. Liu, X.; Yang, J. li; Tong, L. Yan; Zhang, Q.; Li, X. Wei; Chen, J. da.; Chem. Res. Chin. Univ. 2015, 31, 6.

86. Demirkurt, M.; Olcer, Y. A.; Demir, M. M.; Eroglu, A. E.; Anal. Chim. Acta 2018, 1014, 1.

87. Mohammadi, V.; Saraji, M.; Jafari, M. T.; Microchim. Acta 2019, 186, 8.

88. Büttiker, R.; Ebert, J.; Hinderling, C.; Adlhart, C.; CHIMIA International Journal for Chemistry 2011, 65, 3.

89. Wu, K.; Yang, W.; Jiao, Y.; Zhou, C.; J. Mater. Chem. B. 2017, 5, 29.

90. Wu, Y.; Zhang, Y.; Zhang, M.; Liu, F.; Wan, Y.; Huang, Z.; Ye, L.; Zhou, Q.; Shi, Y.; Lu, B.; Food Chem. 2014, 164, 527.

91. Ying, X.; Zhu, X.; Kang, A.; Li, X.; Talanta 2019, 204, 647.

92. Awokoya, K. N.; Moronkola, B. A.; Chigome, S.; Ondigo, D. A.; Tshentu, Z.; Torto, N.; J. Polym. Res. 2013, $20,6$.

93. Beigzadeh, Z.; Golbabaei, F.; Khadem, M.; Omidi, F.; Someah, M. S.; Shahtaheri, S. J.; Macromol. Res. 2020, 28, 390.

94. Abdul-quadir, M.; Ferg, Ernst.; Tshentu, Zenixole.; Ogunlaja, Adeniyi.; RSC Adv. 2018, 8, 8039.

95. Ardekani, R.; Borhani, S.; Rezaei, B.; J. Appl. Polym. Sci. 2018, 136, 47112.

96. Tonglairoum, P.; Chaijaroenluk, W.; Rojanarata, T.; Ngawhirunpat, T.; Akkaramongkolporn, P.; Opanasopit, P.; AAPS PharmSciTech. 2013, 14, 2.

97. Vatta, L. L.; Sanderson, R. D.; Koch, K. R.; Pure Appl. Chem. 2006, 78, 9.

98. Hu, B.; He, M.; Chen, B.; Solid-Phase Extraction 2020, 235, doi:10.1016/B978-0-12-816906-3.00009-1. 
99. Keçili, R.; Büyüktiryaki, S.; Dolak, İ.; Hussain, C. M. In Handbook of Nanomaterials in Analytical Chemistry; Hussain, C. M., ed.; Elsevier: Amsterdam, 2020, pp. 75-95.

100. Lan, T.; Shao, Z.; Wang, J.; Gu, M.; Chem. Eng. J. 2015, $260,818$.

101. Hota, G.; Kumar, B. R.; Ng, W. J.; Ramakrishna, S.; J. Mater. Sci. 2007, $43,1$.

102. Wang, M.; Li, X.; Hua, W.; Shen, L.; Yu, X.; Wang, X.; ACS Appl. Mater. Interfaces 2016, 8,36 .

103. Shariful, M. I.; Sharif, S. B.; Lee, J. J. L.; Habiba, U.; Ang, B. C.; Amalina, M. A.; Carbohydr. Polym. 2017, 157.

104. Haddad, M. Y.; Alharbi, H. F.; J. Appl. Polym. Sci. 2018, 136, 47209.

105. Li, L.; Wang, F.; Lv, Y.; Liu, J.; Zhang, D.; Shao, Z.; Appl. Clay Sci. 2018, 161, 225.

106. Alharbi, H. F.; Haddad, M. Y.; Aijaz, M. O.; Assaifan, A. K.; Karim, M. R.; Coatings 2020, 10, 285.

107. Bajaj, B.; Joh, H.-I.; Jo, S. M.; Park, J. H.; Yi, K. B.; Lee, S.; Appl. Surf. Sci. 2018, 429, 253.

108. Chen, X.; Zhang, F.; Wang, Q.; Han, X.; Li, X.; Liu, J.; Qu, F.; Dalton Trans. 2015, 44, 7.

109. Gao, Q.; Luo, J.; Wang, X.; Gao, C.; Ge, M.; Nanoscale Res. Lett. 2015, 10,1 .

110. Pant, B.; Barakat, N. A. M.; Pant, H. R.; Park, M.; Saud, P. S.; Kim, J.-W.; Kim, H.-Y.; J. Colloid Interface Sci. 2014, 434, 159.

111. Shen, J.; Li, Z.; Wu, Y.; Zhang, B.; Li, F.; Chem. Eng. J. 2015, 264, 48.

112. Zhou, Z.; Liu, L.; Yuan, W.; New J. Chem. 2019, 43, 15823.

113. Zhu, Z.; Xu, Y.; Qi, B.; Zeng, G.; Wu, P.; Liu, G.; Sun, Y.; Environ. Sci: Nano 2017, 4, 2.

114. Liu, Q.; Zhong, L.-B.; Zhao, Q.-B.; Frear, C.; Zheng, Y.-M.; ACS Appl. Mater. Interfaces 2015, 7, 27.

115. Yohai, L.; Giraldo Mejía, H.; Procaccini, R.; Pellice, S.; Laxman Kunjali, K.; Dutta, J.; Uheida, A.; RSC Adv. 2019, 9, 15.

116. Moradi, G.; Dabirian, F.; Mohammadi, P.; Rajabi, L.; Babaei, M.; Shiri, N.; Chem. Eng. Res. Des. 2018, 129, 182.

117. Yin, X.; Zhang, Z.; Ma, H.; Venkateswaran, S.; Hsiao, B. S.; Sep. Purif. Technol. 2020, 242, 116794.

118. Demirci, S.; Celebioglu, A.; Uyar, T.; Carbohydr. Polym. 2014, 113, 200.

119. Du, F.; Sun, L.; Huang, Z.; Chen, Z.; Xu, Z.; Ruan, G.; Zhao, C.; Chemosphere 2019, 239, 124764.
120. Kim, J.; Kang, T.; Kim, H.; Ju Shin, H.; Oh, S.-G.; J. Ind. Eng. Chem. 2019, 77, 273.

121. Bagheri, H.; Roostaie, A.; Anal. Chim. Acta 2015, 863, 20.

122. Bagheri, H.; Khanipour, P.; Asgari, S.; Anal. Chim. Acta 2016, 934, 88.

123. Chigome, S.; Darko, G.; Buttner, U.; Torto, N.; Anal. Methods 2010, 2 , 6.

124. Xu, Q.; Yin, X.; Wu, S.; Wang, M.; Wen, Z.; Gu, Z.; Microchim. Acta 2010, 168,3 .

125. Xu, Q.; Zhang, N.; Yin, X.; Wang, M.; Shen, Y., Xu, S.; Gu, Z.; $J$. Chromatogr. B 2010, 878, 26.

126. Zhang, Y.; Kang, X.; Chen, L.; Pan, C.; Yao, Y.; Gu, Z.-Z.; Anal. Bioanal. Chem. 2008, 391, 6.

127. Kang, X.-J.; Chen, L.-Q.; Wang, Y.; Zhang, Y.-Y.; Gu, Z.-Z.; Biomed. Microdevices 2009, 11, 4.

128. Hajebi, N.; Seidi, S., Ramezani, M.; Manouchehri, M.; New J. Chem. 2020, 44, 14429.

129. Mollahosseini, A.; Alamshahi, M.; Rastegari, M.; J Food Sci Technol. 2020, 57,3792

130. Chen, Z.; Yu, C., Xi, J.; Tang, S., Bao, T.; Zhang, J.; Microchim. Acta. 2019, 186, 6 .

131. Liu, G.; Li, L.; Huang, X.; Zheng, S.; Xu, D., Xu, X.; Zhang, Y.; Lin, H.; Microporous Mesoporous Mater. 2018, 270, 258.

132. Alexiadou, D. K.; Maragou, N. C.; Thomaidis, N. S.; Theodoridis, G. A.; Koupparis, M. A.; J. Sep. Sci. 2008, 31, 12.

133. Wang, L.; Li, J.; Wang, J.; Guo, X.; Wang, X.; Choo, J.; Chen, L.; J. Colloid Interface Sci. 2019, 541, 376.

134. Zhang, Z.; Wang, L.; Liu, X., Zhang, D.; Zhang, L., Li, Q.; RSC Adv. 2015, 5, 105.

135. Zhang, S.; Niu, H., Hu, Z.; Cai, Y.; Shi, Y.; J. Chromatogr. A 2010, 1217, 4757.

136. Yan, Z.; Wu, M.; Hu, B.; Yao, M.; Zhang, L.; Lu, Q.; Pang, J.; J. Chromatogr. A 2018, 1542, 19.

137. Wang, J.; Cai, C.; Zhang, Z.; Li, C.; Liu, R.; Chemosphere 2020, 239, 124833.

138. Patel, S.; Hota, G.; RSC Adv. 2016, 6, 19.

139. Bagheri, H.; Roostaie, A.; J. Chromatogr. A 2014, 1324, 11.

140. Feizbakhsh, A.; Ehteshami, S.; J. AOAC Int. 2017, 100, 1. 